\title{
BASIC REPRESENTATIONS OF ARBITRARY SEMIGROUPS
}

\author{
BY \\ D. B. MCALISTER( $\left.{ }^{1}\right)$
}

In a series of papers, [9], [10], [11], [13], W. D. Munn has considered the problem of constructing all irreducible representations of a semigroup by matrices over a field. In [13] he has shown that the irreducible representations of an inverse semigroup are determined by irreducible representations of Brandt semigroups in the following manner. For any 0 -restricted irreducible representation $\Gamma$ of a semigroup $S=S^{0}$ let

$$
M=\{x \in S: a \in S \mid 0 \text { implies rank } \Gamma(x) \leqq \operatorname{rank} \Gamma(a)\} .
$$

Then $M$ is an ideal of $S$ and $\Gamma$ is uniquely determined by its action on $M$. If $S$ is an inverse semigroup then $\Gamma(M)$ is a Brandt semigroup. Further $M$ has a maximal 0 -restricted Brandt semigroup homomorphic image $M^{*}$ and there is a unique irreducible representation $\Gamma^{*}$ of $M^{*}$ such that the diagram

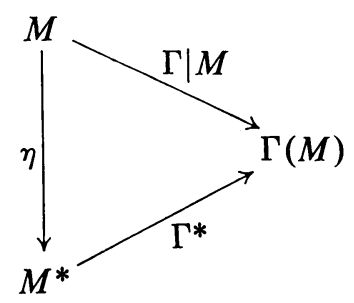

commutes, where $\eta$ is the natural homomorphism of $M$ onto $M^{*}$. Thus $\Gamma$ is uniquely determined by the irreducible representation $\Gamma^{*}$ of the Brandt semigroup $M^{*}$.

In [6], the present author generalised the method of [13] to show that, for certain types of semigroups, in particular for regular semigroups and periodic semigroups, the irreducible representations are determined by those of associated completely 0 -simple semigroups. If $S$ is a semigroup of one of these types and $\Gamma$ is an irreducible 0-restricted representation of $S$ then $\Gamma(M)$ is completely 0 -simple and $M$ has a maximal 0-restricted completely 0 -simple homomorphic image $M^{*}$. Further $\Gamma$ induces a unique representation $\Gamma^{*}$ of $M^{*}$ such that $(0.1)$ commutes. Thus $\Gamma$ is uniquely determined by the irreducible representation $\Gamma^{*}$ of the completely 0 -simple semigroup $M^{*}$. If $S$ is an entirely arbitrary semigroup however $\Gamma(M)$

Received by the editors March 13, 1967 and, in revised form, August 11, 1967.

( $\left.{ }^{1}\right)$ This research was supported by a grant from the National Science Foundation. 
need not be completely 0 -simple nor need $M$ have a maximal 0 -restricted completely 0 -simple homomorphic image. (For example the mapping $x^{n} \rightarrow n, 0 \rightarrow 0$ is an irreducible 0 -restricted representation of the infinite cyclic semigroup, with zero adjoined, $T$, of degree one over the rationals, thus $M=T$ is not completely 0 -simple; further $T$ has no maximal completely 0 -simple homomorphic image.) Hence the methods of [13], [6] do not suffice to give a satisfactory description of the irreducible representations of arbitrary semigroups.

In this paper we use Theorem 1.5 of [7] and the result in italics below (a corollary to Theorem 3.7), which is of independent interest, to show, in Theorèm 6.4, that, although the techniques of [13], [6] are inapplicable, the irreducible representations of an arbitrary semigroup are also determined by those of certain associated completely 0 -simple semigroups which depend only on $S$.

Let $H=H^{0}$ be a semigroup of linear transformations of a finite dimensional vector space $\mathscr{V}$. Suppose that all nonzero elements of $H$ have the same rank and that $H$ has no nonzero left or right annihilators. Then $H$ is contained in a completely 0 -simple semigroup of linear transformations of $\mathscr{V}$.

The techniques used to prove Theorem 6.4 may be applied to a type of matrix representation which is more general than an irreducible representation; we call this type of representation basic. A representation $\Gamma$ of a semigroup $S=S^{0}$ by linear transformations of a finite dimensional vector space $\mathscr{V}$ is basic if, for each ideal $N$ of $S$ such that $\Gamma(N) \neq 0, \mathscr{V} \Gamma(N)$ spans $\mathscr{V}$ and $\Gamma(N)$ annihilates no nonzero subspace of $\mathscr{V}$. Thus every proper representation of a group is basic. Further Theorem 3.12 shows that, for a completely C-simple semigroup, the basic representations defined here coincide with those defined by A. H. Clifford in [1], [2]. The main theorem of $\S 6$ gives an explicit method for constructing the basic representations of an arbitrary semigroup modulo the basic representations of completely 0 -simple semigroups (by Theorem 3.12, the latter may be regarded as well known).

The prevalence of basic representations of a semigroup depends on its "grouplike" character. In fact Theorem 2.4 shows that every proper representation of a finite semigroup $S=S^{0}$, over a field $\Phi$, is basic if and only if $S$ is 0 -simple and the contracted semigroup algebra $\Phi_{0}[S]$ of $S$ is isomorphic to a full matrix algebra over a group algebra. In $\S 7$, sufficient conditions are given on a semigroup $S=S^{0}$ in order that each representation of $S$, over a field $\Phi$, should decompose into basic representations; if this is the case, we say that $S$ is quasi-simple over $\Phi$. These conditions permit us to prove the result, which can be deduced from G. B. Preston [14], that an inverse semigroup is quasi-simple over every field. (In a sequel to this paper, [8], we consider finite semigroups which are quasi-simple over a field. The results of that paper demonstrate further the relationship between the grouplike character of a finite semigroup and the prevalence of its basic representations.)

The basic representations $\Gamma$ of a semigroup $S=S^{0}$ are the most general representations of $S$ with the property that $\Gamma(S)$ is the extension, in a natural manner, 
of the ideal consisting of 0 and the elements of minimal nonzero rank. This ideal is homogeneous and in $\$ 3,4$ we consider in some detail homogeneous semigroups of matrices and representations whose images are such semigroups. The material in $\S 4$ is of a technical nature and is required for the proofs of the results in $\$ \S 6,7$. Using the results obtained in $\$ 4$, we are also able to give, in $\$ 5$, a method for constructing all representations of an arbitrary 0 -simple semigroup (again moduio the theory of [1], [2]).

Finally, I should like to express my gratitude to A. H. Clifford for reading a rough draft of this paper and for many helpful suggestions concerning the presentation of the material contained here.

1. Preliminaries. Except for those concepts explicitly defined here, we shall use the terminology and notation of Clifford and Preston [3]. We shall also assume familiarity with the results of [3], $\$ 5.1-5.4$, and with [7], $\$ \$ 1$ and 2.

The following lemma from [7] characterises completely 0-simple semigroups. This characterisation and the conditions which it introduces will play an important part in the theory contained in this paper.

LEMMA 1.1. Let $S=S^{0}$ be a semigroup, then $S^{0}$ is completely 0 -simple if and only if the following conditions are satisfied:

$\mathrm{C}_{1}$ : if, for $a, x, b \in S, a x b=0$ then $a x=0$ or $x b=0$;

$\mathrm{C}_{2}$ : if, for $a, b \in S, a S b=0$ then $a=0$ or $b=0$;

$\mathrm{C}_{3}$ : if, for $a, b, x, y \in S, a x=b x \neq 0$ and $y a=y b \neq 0$ then $a=b$;

$\mathrm{C}_{4}$ : for each $a \in S$ such that $a^{2} \neq 0$, there exists a unique element $a^{-1} \in S$ such that

$$
a=a a^{-1} a, \quad a^{-1}=a^{-1} a a^{-1}, \quad a a^{-1}=a^{-1} a .
$$

A semigroup $S=S^{0}$ which obeys $\mathrm{C}_{1}$ is said to be categorical at zero; if $S$ obeys $\mathrm{C}_{2}$ it is said to be indecomposable at zero; if $S$ obeys both $\mathrm{C}_{1}$ and $\mathrm{C}_{2}$ it is said to be 0-primary. If $S$ obeys $\mathrm{C}_{3}$ it is said to be weakly 0 -cancellative while $\mathrm{C}_{4}$ is equivalent to the assertion that each element of $S$ whose square is nonzero belongs to a subgroup of $S$.

The main tool in this paper is Theorem 1.5 of [7] and a generalisation thereof (Theorem 1.6). In order to apply Theorem 1.5 of [7], we need some information concerning the structure of the completely 0 -simple semigroup $\mathscr{C}(S)$ whose existence is asserted in that theorem. The major part of this section is devoted to obtaining this information and to proving Theorem 1.6.

Let $S=S^{0}$ be a completely 0 -simple semigroup and let $\mathscr{H}$ denote the usual Green's equivalence on $S$; [3, p. 48]. Then $\mathscr{H}$ is a congruence on $S$ and $S / \mathscr{H}$ is a rectangular 0-band; [4, p. 69]. In particular, if $a, b, x, y \in S$ then

$$
\text { (i) if } a x b \neq 0, a y b \neq 0 \text { then }(a x b, a y b) \in \mathscr{H} \text {; }
$$

(ii) if axa $\neq 0$, then $(a, a x a) \in \mathscr{H}$. 
These results are easily deduced from the fact that $S$ is isomorphic to a regular Rees matrix semigroup $\mathscr{M}^{0}(G ; I, \Lambda ; P)$; for here, $((x ; i, \lambda),(y ; j, \mu)) \in \mathscr{H}$ if and only if $i=j$ and $\lambda=\mu$.

LEMMA 1.2. Let $S=S^{0}$ be a 0 -primary semigroup and let $N$ be a nonzero ideal of $S$. Then each $\mathscr{H}$-class of $\mathscr{C}(S)$ contains an element of $N \eta$, where $\eta$ is the natural 0 restricted homomorphism of $S$ into $\mathscr{C}(S)$.

Proof. We show first that the lemma is true for $N=S$. As in [7, Theorem 1.5], $\mathscr{C}(S)=\bigcup\left\{S_{n}: n \geqq 0\right\}$ and we use induction on $n$ to show that, if $x \in S_{n}$, then $(x, s \eta) \in \mathscr{H}$ for some $s \in S$. Since $S_{0}=S$, this is immediate if $n=0$.

Suppose that the result is true for $n$ and let $x \in S_{n+1} \mid S_{n}$. Then $x$ is a product of elements of $S_{n}$ and their inverses and hence there exist $u, v \in S_{n}, y \in S_{n+1}$ such that $x=u y v$. By induction hypothesis, there exist $s, t \in S$ such that $(u, s \eta) \in \mathscr{H}$ and $(v, t \eta) \in \mathscr{H}$. Since $\mathscr{H}$ is a congruence on $\mathscr{C}(S)$, this implies $(x, s \eta y(t \eta)) \in \mathscr{H}$. Since $x \neq 0$, neither of $s, t$ is zero and thus, because $S$ is indecomposable at zero, there exists $w \in S$ such that $s w t \neq 0$. Since $\mathscr{C}(S)$ is completely 0 -simple, it follows from (1.1), (i), that $(s \eta y(t \eta),(s w t) \eta) \in \mathscr{H}$ and hence $(x,(s w t) \eta) \in \mathscr{H}$. But $s w t \in S$ so that the result is true for $x \in S_{n+1}$. Hence, by induction, it is true for any $x \in \mathscr{C}(S)$.

Let $s \in S \mid 0$; then, since $S$ is indecomposable at zero, there exists $m \in N$ such that $s m s \neq 0$ (let $n \in N \mid 0$, then $s S n \neq 0, n S s \neq 0$ and so $0 \neq s S n \cdot S \cdot n S s \subseteq s N s$ ). Then $s m s \in N$ and, by (1.1), (ii), $(s \eta,(s m s) \eta) \in \mathscr{H}$. Thus, since each $\mathscr{H}$-class of $\mathscr{C}(S)$ contains an element of $S \eta$, each $\mathscr{H}$-class contains an element of $N \eta$.

COROllary 1. Let $S=S^{0}$ be a 0 -primary semigroup. Then $\mathscr{C}(S) / \mathscr{H}$ is the maximal rectangular 0-band homomorphic image of $S$.

Proof. This is almost immediate from Lemma 1.2 and [7, Theorem 1.5].

COROLlary 2. Let $S=S^{0}$ be a 0 -primary semigroup and let $N$ be a nonzero ideal of $S$. For each $x \in \mathscr{C}(S)$ there exist $u, v \in N, y \in \mathscr{C}(S)$ such that $x=(u \eta) y(v \eta)$.

Proof. If $x \neq 0$ then there exist idempotents $e, f \in \mathscr{C}(S)$ such that $x=e x f$. By Lemma 1.2 , there exist $u, v \in N$ such that $(u \eta, e) \in \mathscr{H},(v \eta, f) \in \mathscr{H}$. These imply $e=(u \eta)(u \eta)^{-1}, f=(v \eta)(v \eta)^{-1}$ thus, if $y=(u \eta)^{-1} x(v \eta)^{-1}$ we have $x=(u \eta) y(v \eta)$ as required.

A semigroup $S=S^{0}$ is said to be a 0 -direct union of a family $\left\{S_{i}: i \in I\right\}$ of its subsemigroups if

(a) $S=\bigcup\left\{S_{i}: i \in I\right\}$;

(b) if $i \neq j$ then $S_{i} \cap S_{j}=0=S_{i} S_{j}$.

If (a) and (b) hold, we write $S=0\left\{S_{i}: i \in I\right\}$. Note that conditions (a) and (b) together imply that each $S_{i}, i \in I$, is an ideal of $S$.

Preston [14] and Lallement and Petrich [5] have shown that a semigroup $S=S^{0}$ is a 0 -direct union of completely 0 -simple semigroups if and only if it is regular and each idempotent is primitive; following Preston [14], we say that such a semigroup 
is primitive regular or p-regular. Lallement and Petrich have also shown, [5, Corollary 5.13], that a semigroup $S=S^{0}$ is a 0 -direct union of 0 -primary semigroups if and only if $S$ is categorical at zero and obeys

$$
\mathrm{C}_{2}^{\prime}: \text { for } a \in S \text {, if } a S a=0 \text { then } a=0 .
$$

The following lemma, whose proof is straightforward, shows that $\mathbf{C}_{2}^{\prime}$ is equivalent to the condition that $S$ has no nonzero nilpotent ideals.

LEMMA 1.3. A semigroup $S=S^{0}$ is without nonzero nilpotent ideals if and only if it obeys $\mathrm{C}_{2}^{\prime}$; $S$ is semisimple if and only if each nontrivial homomorphic image obeys $\mathrm{C}_{2}^{\prime}$.

The proof of the following lemma is routine; it is thus omitted.

LEMmA 1.4. Let $S=0\left\{S_{i}: i \in I\right\}$ and $T=0\left\{T_{\alpha}: \alpha \in A\right\}$ be 0-direct unions of 0 -primary semigroups. Let $\theta$ be a 0 -restricted homomorphism of $S$ into $T$. Then there exists a mapping $\varphi: I \rightarrow A$ and, for each $i \in I$, a 0 -restricted homomorphism $\theta_{i}$ of $S_{i}$ into $T_{i \varphi}$ such that, for each $a \in S_{i}$,

$$
a \theta=a \theta_{i} .
$$

Conversely, given $\varphi: I \rightarrow A$ and $\theta_{i}: S_{i} \rightarrow T_{i}$, the mapping $\theta$ defined by (1.2) is a 0 -restricted homomorphism of $S$ into $T$.

If $S=S^{0}$ is a subsemigroup of a $p$-regular semigroup $T$ then there need not be a minimum $p$-regular subsemigroup of $T$ which contains $S$. However, if $S$ is indecomposable at zero and $T$ is completely 0 -simple, it follows from the results of [7] that there is a minimum completely 0 -simple subsemigroup of $T$ containing $S$. Suppose now that $S$ is the 0 -direct union of a family $\left\{S_{i}: i \in I\right\}$ of 0 -primary semigroups and that $S$ is a subsemigroup of the p-regular semigroup $T=0\left\{T_{\alpha}: \alpha \in A\right\}$. Then, for each $i \in I$, there exists $i \varphi \in A$ such that $S_{i} \subseteq T_{i \varphi}$. Hence there exists a completely 0 -simple subsemigroup $\bar{S}_{i}$ of $T_{i \varphi}$ generated by $S_{i} ; \bar{S}_{i}$ is clearly the completely 0 -simple subsemigroup of $T$ generated by $S_{i}$.

Lemma 1.5. Let $S=S^{0}$ be the 0-direct union of 0-primary semigroups $\left\{S_{i}: i \in I\right\}$. Suppose that $S$ is contained in a p-regular semigroup $T$ and, for each $i \in I$, let $\bar{S}_{i}$ be the completely 0-simple subsemigroup of $T$ generated by $S_{i}$. Then, if $i \neq j, \bar{S}_{i} \cap \bar{S}_{j}$ $=0=\bar{S}_{i} \bar{S}_{j}$ and $\bar{S}=0\left\{\bar{S}_{i}: i \in I\right\}$ is the minimum p-regular subsemigroup of $T$ which contains $S$.

Proof. Clearly $\bigcup\left\{\bar{S}_{i}: i \in I\right\}$ is contained in any $p$-regular subsemigroup of $T$ which contains $S$ so that we need only show $\bar{S}_{i} \cap \bar{S}_{j}=\bar{S}_{i} \bar{S}_{j}=0$ if $i \neq j$. If $S_{i}, S_{j}$ belong to different completely 0 -simple components of $T$, this is clear so we may suppose that $S_{i}, S_{j}$ belong to a completely 0-simple subsemigroup of $T$.

$\bar{S}_{j}=\bigcup\left\{S_{j n}: n \geqq 0\right\}$ where $S_{j}=S_{j 0}$ and $S_{j n+1}$ is generated by the elements of $S_{j n}$ and their inverses. We use induction on $n$ to show that, if $i \neq j$,

$$
S_{i} \cap \bar{S}_{j}=0=S_{i} \bar{S}_{j}=\bar{S}_{j} S_{i} ;
$$

a similar argument then proves the stronger result. 
It is clear that $S_{i} \cap S_{j m}=0=S_{i} S_{j m}=S_{j m} S_{i}$ when $m=0$, so suppose that it is true with $m=n$, and let $x \in S_{n+1}$. Then, as in Lemma 1.2, $x=u y v$ where $u, v \in S_{j n}$. Hence, by induction hypothesis,

$$
S_{i} x=S_{i} u y v=0=u y v S_{i}=x S_{i} .
$$

Further, since $S_{i}$ is indecomposable at zero, it is impossible that $x \in S_{i} \cap S_{j n+1} \mid 0$. Thus, by induction, (1.3) is true.

Theorem 1.6 now follows easily from Lemmas 1.4, 1.5 and [7, Theorem 1.5].

THEOREM 1.6. Let $S=S^{0}$ be a semigroup which is categorical at zero and has no nonzero nilpotent ideals. Then there is a p-regular semigroup $\mathscr{P}(S)$ and a 0 -restricted homomorphism $\eta$ of $S$ into $\mathscr{P}(S)$ such that

(a) $\mathscr{P}(S)$ is generated by $S \eta$ as a p-regular semigroup;

(b) given any 0-restricted homomorphism $\theta$ of $S$ into a p-regular semigroup $T$ there exists a unique 0-restricted homomorphism $\varphi$ of $\mathscr{P}(S)$ into $T$ such that the diagram

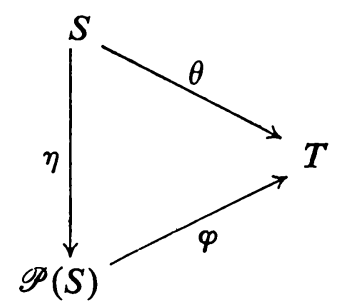

commutes;

(c) if $S=0\left\{S_{i}: i \in I\right\}$ where each $S$ is 0-primary, then $\mathscr{P}(S)=0\left\{\mathscr{C}\left(S_{i}\right): i \in I\right\}$ where $\mathscr{C}\left(S_{i}\right)$ is the unique completely 0-simple semigroup determined by $S_{i}$ as in [7, Theorem 1.5];

(d) if $T$ is generated, as a p-regular semigroup, by $S \theta$, then $\varphi$ is onto $T$.

2. Basic representations (Introduction). By a representation of a semigroup $S=S^{0}$, of degree $n$ over a field $\Phi$, we mean a homomorphism $\Gamma$ of $S=S^{0}$ into the algebra $[\Phi]_{n}$ of $n \times n$ matrices over $\Phi$, or, equivalently, into the algebra $\mathscr{L} \mathscr{T}(\mathscr{V})$ of linear transformations of an $n$ dimensional vector space $\mathscr{V}$ over $\Phi$, such that $\Gamma(0)=0$. A representation $\Gamma$ of $S=S^{0}$ by linear transformations of the finite dimensional vector space $\mathscr{V}$ over $\Phi$, is basic if, for each ideal $N$ of $S$ such that $\Gamma(N) \neq 0$,

(i) $\mathscr{U} \Gamma(N) \neq 0$ whenever $\mathscr{U}$ is a nonzero subspace of $\mathscr{V}$;

(ii) $[\mathscr{V} \Gamma(N)]=\mathscr{V}$;

where, for any nonempty subset $\mathscr{X}$ of $\mathscr{V},[\mathscr{X}]$ is the subspace of $\mathscr{V}$ spanned by $\mathscr{X}$. 
There is a natural one-to-one correspondence between the representations of a semigroup $S=S^{0}$ over $\Phi$ and those of the contracted algebra $\Phi_{0}[S]$ of $S$ over $\Phi$. This correspondence preserves the equivalence, decomposition and reduction of representations. In general, it seems difficult to define representations of an arbitrary ring so that, when we consider semigroup algebras, the representations under consideration are just those induced by the basic representations of the semigroup. (For example, if we define an $R$-module $A$ to be "basic" if the analog of (2.1) holds for all ideals $N$ of $R$ such that $A \cdot N \neq 0$, the radical induced on $R$ by the "basic" $R$-modules lies between the prime radical and the Jacobson radical; if $R$ is commutative, it is just the prime radical. Hence, for a finite semigroup, the "basic" representations are completely reducible; this is, in general, not the case for basic representations.) As far as 0-simple semigroups are concerned however, one can easily characterise the basic representations directly in terms of the contracted algebra. At the end of this section, we shall indicate how this approach can be used to give necessary and sufficient conditions on a finite 0 -simple semigroup in order that every proper representation should be basic; we shall not prove the result in detail as equivalent results are given in [8].

In the case of arbitrary semigroups, it is more natural to approach the problem of constructing the basic representations directly through the semigroup rather than through the contracted semigroup algebra. Theorem 2.1 gives a characterisation of basic representations of an arbitrary semigroup in terms of basic representations of completely 0 -simple semigroups (by Theorem 3.12, the latter can be regarded as well known). Theorem 2.1 is an immediate corollary to Theorem 6.2 ; we state it here since it motivates much of the material in $\$ \$ 3$ and 4.

Let $\Gamma$ be a representation of a semigroup $S=S^{0}$, of degree $n$ over a field $\Phi$. Then, following Munn, [13], we define

$$
\begin{aligned}
& V(\Gamma)=\{x \in S: \Gamma(x)=0\} ; \\
& r(\Gamma)=\min \{\operatorname{rank} \Gamma(x): x \in S \backslash V(\Gamma)\} ; \\
& M(\Gamma)=\{x \in S: \operatorname{rank} \Gamma(x)=r\} \cup V(\Gamma) .
\end{aligned}
$$

Clearly $V(\Gamma)$ and $M(\Gamma)$ are ideals of $S$ and $V(\Gamma) \subset M(\Gamma)$ unless $\Gamma$ is the null representation of $S ; V(\Gamma)$ is called the vanishing ideal of $\Gamma$. If there is no risk of ambiguity, we shall write $V$ for $V(\Gamma)$ and $M$ for $M(\Gamma)$; we shall also reserve the letters $V$ and $M$ for the $V(\Gamma)$ and $M(\Gamma)$ of an associated representation.

A pair $(U, N)$ of ideals of a semigroup $S=S^{0}$, with $U \subset N$, is a primary pair if

(a) $S / U$ is indecomposable at zero;

(b) $N / U$ is categorical at zero;

thus $S$ is 0 -primary if and only if $(0, S)$ is a primary pair. Note that, since $N / U$ is an ideal of $S / U$ and the latter is indecomposable at zero, $N / U$ is 0 -primary.

THEOREM 2.1. Let $S=S^{0}$ be a semigroup and let $\Gamma$ be a representation of $S$ of degree $n$ over a field $\Phi$. Then $\Gamma$ is basic if and only if

(a) $(V, M)$ is a primary pair; 
(b) $\Gamma$ induces a (unique) representation $\Gamma^{*}$ of $\mathscr{C}(M / V)$ into $[\Phi]_{n}$ such that the diagram

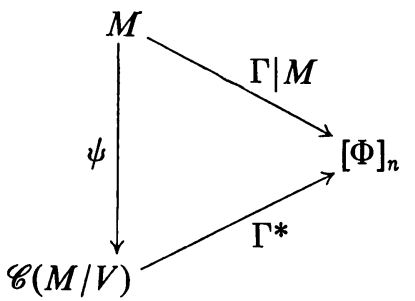

commutes, where $\psi=f_{\eta}$ with $f$ the natural homomorphism of $M$ onto $M / V$ and $\eta$ the natural 0-restricted homomorphism of $M / V$ into $\mathscr{C}(M / V)$.

If $S=S^{0}$ is a 0 -simple semigroup, then it is immediate that a nonnull representation $\Gamma$ of $S$ over $\Phi$ is basic if and only if

(i) $\mathscr{U} \Gamma\left(\Phi_{0}[S]\right) \neq 0$ whenever $\mathscr{U}$ is a nonzero subspace of $\mathscr{V}$;

(ii) $\left[\mathscr{V} \Gamma\left(\Phi_{0}[S]\right)\right]=\mathscr{V}$.

With this in mind, we define an $R$-module $A$ ( $R$ any ring) to be weakly basic if

(i) for each nonzero $a \in A, a \cdot R \neq 0$;

(ii) $[A \cdot R]=A$;

where $[\mathscr{X}$ ] denotes the subgroup of $A$ generated by the nonvoid subset $\mathscr{X}$. The weak basic radical $\mathscr{W}(R)$ is $\{x \in R: A \cdot x=0$ for each weakly basic $R$-module $A\}$.

THEOREM 2.2. Let $R$ be any ring that is generated (as a group) by $R^{2}$. Then

$$
\mathscr{W}(R)=\{x \in R: R x R=0\} .
$$

Proof. It is easy to show that, if $x \in B(R)=\{x \in R: R x R=0\}$, then $A \cdot x=0$ for each weakly basic $R$-module $A$. Conversely, given the hypothesis on $R$, one can show that $R / B(R)$ is a weakly basic $R$-module and that $R / B(R) \cdot x=0$ if and only if $x \in B(R)$.

Since $S^{2}=S$ for any (finite) 0 -simple semigroup, $\Phi_{0}[S]$ is generated (as a group) by $\Phi_{0}[S]^{2}$ and thus the basic radical

$$
\mathscr{B}_{\Phi}(S)=\left\{x \in \Phi_{0}[S]: \Gamma(x)=0 \text { for all basic representations of } S \text { over } \Phi\right\}
$$

of $\Phi_{0}[S]$ is given by Theorem 2.2. In fact, using the results of $[3, \S 5.2]$, we have the following theorem.

THEOREM 2.3. Let $S=\mathscr{M}^{0}(G ; m, n ; P)$ be a finite 0-simple semigroup and let $\Phi$ be a field. Then $\mathscr{B}_{\Phi}(S)=\left\{x \in \Phi_{0}[S]: S x S=0\right\}$.

Further, the following are equivalent

(1) $\mathscr{B}_{\Phi}(S)=0$;

(2) $P$ is invertible over $\Phi[G]$;

(3) $\Phi_{0}[S]$ has an identity; 
(4) $m=n$ and $\Phi_{0}[S]$ is isomorphic to the algebra of all $n \times n$ matrices over $\Phi[G]$;

(5) $S$ is quasi-simple over $\Phi$; that is, each nonnull indecomposable representation of $S$ over $\Phi$ is basic;

(6) each proper representation of $S$ over $\Phi$ is basic.

RemarK. Lallement and Petrich, [5, Theorem 2.1], have shown that, in the Munn algebra $\mathfrak{B}$ isomorphic to $\Phi_{0}[S]$ see [3, p. 162], the (Jacobson) radical is given by $\operatorname{rad} \mathfrak{B}=\{X \in \mathfrak{B}: P X P$ is an $n \times m$ matrix over $\operatorname{rad} \Phi[G]\}$.

Their proof of this result uses the structure of irreducible representations of a completely 0 -simple semigroup. It can, however, be adapted to yield the characterisation of $\mathscr{B}_{\Phi}(S)$ given in Theorem 2.3.

Theorem 2.3 characterises those finite 0 -simple semigroups with the property that each proper representation, over a field $\Phi$, is basic. The following theorem shows that it essentially characterises all finite semigroups with this property. To prove the theorem, we make use of the fact that, for each ideal $N$ of a semigroup $S=S^{0}$, there is at least one representation of $S$, over a field $\Phi$, with vanishing ideal $N$. (For example, let $\eta$ be a one-to-one mapping of $S^{1} \backslash N$ onto a basis for a $\left|S^{1} \backslash N\right|$-dimensional vector space $\mathscr{V}$ over $\Phi$. For each $a \in S, x \in S^{1} \backslash N$ define

$$
\begin{aligned}
x \eta \Gamma(a) & =(x a) \eta & & \text { if } x a \notin N, \\
& =0 & & \text { otherwise, }
\end{aligned}
$$

and extend $\Gamma(a)$ linearly to all of $\mathscr{V}$. Then the mapping $\Gamma: a \rightarrow \Gamma(a)$ is a representation of $S$ with vanishing ideal $N$; hence the proper part of $\Gamma$ has vanishing ideal $N$.)

THEOREM 2.4. Let $S=S^{0}$ be a finite semigroup and let $\Phi$ be a field. Then each proper representation of $S$ over $\Phi$ is basic if and only if $S$ is 0-simple and satisfies the conditions (1) through (6) of Theorem 2.3.

Proof. Suppose that every proper representation of $S$ is basic. If $S$ is not 0 -simple then $S^{2}=0$ or $S$ has a nonzero proper ideal $N$. In the former case, it is immediate from Theorem 2.1 that $S$ can admit no 0 -restricted basic representation. Hence $S^{2} \neq 0$.

If $N$ is a nonzero proper ideal of $S$, let $\Gamma_{1}$ be a proper 0-restricted representation of $S$ with underlying vector space $\mathscr{V}_{1}$ and let $\Gamma_{2}$ be a proper representation of $S$ with vanishing ideal $N$ and underlying vector space $\mathscr{V}_{2}$. Then $\Gamma=\Gamma_{1} \oplus \Gamma_{2}$ is a proper representation of $S$ with underlying vector space $\mathscr{V}=\mathscr{V}_{1} \oplus \mathscr{V}_{2}$. However

$$
\mathscr{V} \Gamma(N)=\mathscr{V}_{1} \Gamma_{1}(N)+\mathscr{V}_{2} \Gamma_{2}(N)=\mathscr{V}_{1} \Gamma_{1}(N) \subseteq \mathscr{V}_{1}
$$

Hence $\Gamma$ is not basic.

Thus $S$ is 0 -simple if each of its proper representations is basic and so, from Theorem 2.3, obeys the conditions (1) through (6). Conversely, of course, Theorem 2.3 shows the sufficiency of the conditions. 
3. Homogeneous semigroups of linear transformations. Throughout this section, let $\mathscr{V}$ be a vector space of dimension $n$ over a field $\Phi$. Let $\alpha \in \mathscr{L} \mathscr{T}(\mathscr{V})$ and denote by $\mathscr{V} \alpha$ the range of $\alpha$, by $N \alpha$ the null space of $\alpha$ and by $|\alpha|$ the rank of $\alpha=\operatorname{dim} \mathscr{V} \alpha$.

A subsemigroup $H=H^{0}$ of $\mathscr{L} \mathscr{T}(\mathscr{V})$ is said to be homogeneous if all nonzero members of $H$ have the same rank; clearly any 0-simple subsemigroup of $\mathscr{L} \mathscr{T}(\mathscr{V})$ is homogeneous. In this section we shall obtain some properties of homogeneous semigroups which will be needed later; certain of these are interesting in their own right.

LEMMA 3.1 [13]. Let $H=H^{0}$ be a homogeneous semigroup of linear transformations of a finite dimensional vector space; then $H$ is categorical at zero and weakly 0 -cancellative.

COROLlaRY. Let $\Gamma$ be a representation of a semigroup $S=S^{0}$ of degree $n$ over a field $\Phi$. Then $\Gamma(M)$ is categorical at zero and weakly 0-cancellative.

Proof. From the definition of $M, \Gamma(M)$ is clearly homogeneous.

The following lemma is essentially given in [3, Exercise 6(f), p. 57].

Lemma 3.2. Let $\alpha \in \mathscr{L} \mathscr{T}(\mathscr{V})$ be such that $\left|\alpha^{2}\right|=|\alpha|$. Then $\mathscr{V}=\mathscr{V} \alpha \oplus N \alpha$ and $\alpha=\varepsilon \alpha^{\prime}$ where $\varepsilon$ is the projection of $\mathscr{V}$ on $\mathscr{V} \alpha$ and $\alpha^{\prime}$ is a nonsingular linear transformation of $\mathscr{V}_{\alpha}$ onto itself.

COROLlaRY [3]. If $|\alpha|=\left|\alpha^{2}\right|$ then there exists a unique $\alpha^{-1} \in \mathscr{L} \mathscr{T}(\mathscr{V})$ such that

$$
\alpha=\alpha \alpha^{-1} \alpha, \quad \alpha^{-1}=\alpha^{-1} \alpha \alpha^{-1}, \quad \alpha \alpha^{-1}=\alpha^{-1} \alpha ; \quad\left(\alpha^{-1}=\varepsilon\left(\alpha^{\prime}\right)^{-1}\right) .
$$

LEMMA 3.3. If $|\alpha|=\left|\alpha^{2}\right|$ and $\mathscr{U} \alpha \subseteq \mathscr{U}$ where $\mathscr{U}$ is a subspace of $\mathscr{V}$ then $\mathscr{U} \alpha^{-1} \subseteq \mathscr{U}$; if $\mathscr{Y}$ is a subspace of $\mathscr{V}$ and $\mathscr{U} \alpha \subseteq \mathscr{Y}$ where $\mathscr{Y} \alpha \subseteq \mathscr{Y}$ then $\mathscr{U} \alpha^{-1} \subseteq \mathscr{Y}$.

Proof. We use Lemma 3.2 and its corollary. Let $\mathscr{U}_{1}=\mathscr{U} \alpha \subseteq \mathscr{U}$ and let $\mathscr{U}_{2}$ be such that $\mathscr{V}_{\alpha}=\mathscr{U}_{1} \oplus \mathscr{U}_{2} ;$ thus $\mathscr{V}=\mathscr{U}_{1} \oplus \mathscr{U}_{2} \oplus N \alpha$. Since $\mathscr{U}_{1} \subseteq \mathscr{V}_{\alpha}, \mathscr{U}_{1} \alpha^{\prime}$ $=\mathscr{U}_{1} \alpha \subseteq \mathscr{U}_{1}$ and hence, since $\alpha^{\prime}$ is nonsingular from $\mathscr{V}_{\alpha}$ onto $\mathscr{V} \alpha, \mathscr{U}_{1} \alpha^{\prime}=\mathscr{U}_{1}$. Thus $(\mathscr{U} \varepsilon) \alpha^{\prime}=\mathscr{U}_{1}=\mathscr{U}_{1} \varepsilon \subseteq \mathscr{U}_{\varepsilon}$. Since $\mathscr{U} \varepsilon \subseteq \mathscr{V} \alpha$, this implies $(\mathscr{U} \varepsilon) \alpha^{\prime}=\mathscr{U}_{\varepsilon}$; that is, $\mathscr{U}_{1}=\mathscr{U} \varepsilon$. But this means

$$
\mathscr{U}_{\alpha}{ }^{-1}=\mathscr{U} \varepsilon\left(\alpha^{\prime}\right)^{-1}=\mathscr{U}_{1}\left(\alpha^{\prime}\right)^{-1}=\mathscr{U}_{1} \alpha^{\prime}\left(\alpha^{\prime}\right)^{-1}=\mathscr{U}_{1} \varepsilon=\mathscr{U}_{1} .
$$

Thus we have the first assertion of the lemma.

For the second part, let $\mathscr{U} \alpha \subseteq \mathscr{Y}$; then $\mathscr{U} \alpha^{-1}=\mathscr{U} \alpha \alpha^{-2} \subseteq \mathscr{Y} \alpha^{-2}$. But, by the first part, $\mathscr{Y} \alpha^{-2} \subseteq \mathscr{Y}$.

Let $H=H^{0}$ be a homogeneous subsemigroup of $\mathscr{L} \mathscr{T}(\mathscr{V})$ and let

$$
\mathscr{F}(H)=\left\{\alpha \in \mathscr{L} \mathscr{T}(\mathscr{V}): \mathscr{V}_{\alpha}=\mathscr{V}_{\sigma}, N \alpha=N \tau \text { for some } \sigma, \tau \in H\right\} .
$$

Then $\mathscr{F}(H)$ is called the fill-out of $H$. The reason for this name is explained by the following considerations. In $\mathscr{L} \mathscr{T}(\mathscr{V})$ define $\alpha \sim \beta$ if and only if $\mathscr{V} \alpha=\mathscr{V} \beta$ and $N \alpha=N \beta$. Then $\sim$ is an equivalence on $\mathscr{L} \mathscr{T}(\mathscr{V})$ which coincides with the Green 
equivalence $\mathscr{H}$. If we imagine $\mathscr{L} \mathscr{T}(\mathscr{V})$ arranged in the familiar egg box pattern (cf. [3]), then $\mathscr{F}(H)$ is obtained from $H$ by filling out the $\mathscr{H}$-classes of $\mathscr{L} \mathscr{T}(\mathscr{V})$ which contain elements of $H$ and then filling out the corners of all subrectangles whose sides contain these $\mathscr{H}$-classes.

LEMMA 3.4. Let $H=H^{0}$ be a homogeneous subsemigroup of $\mathscr{L} \mathscr{T}(\mathscr{V})$ and let $\alpha, \beta \in H$. Then either $\mathscr{V} \alpha \cap N \beta=0$ or $\mathscr{V} \alpha \subseteq N \beta$.

Proof. We have either $|\alpha \beta|=|\beta|$ or $|\alpha \beta|=0$. In the latter case, we clearly have $\mathscr{V}_{\alpha} \subseteq N \beta$. In the former case, since $\mathscr{V} \alpha \beta \subseteq \mathscr{V} \beta$, we must have $\mathscr{V} \alpha \beta=\mathscr{V} \beta$ and hence, since $|\alpha|=|\beta|, \mathscr{V}_{\alpha} \cap N \beta=0$.

LEMMA 3.5. Let $H=H^{0}$ be a homogeneous subsemigroup of $\mathscr{L} \mathscr{T}(\mathscr{V})$. Then $\mathscr{F}(H)$ is a homogenous subsemigroup of $\mathscr{L} \mathscr{T}(\mathscr{V})$.

Proof. Let $\alpha, \beta \in \mathscr{F}(H)$; then $\mathscr{V} \alpha=\mathscr{V} \sigma, N \beta=N \tau$ for some $\sigma, \tau \in H$. In particular, $\mathscr{V}_{\alpha} \cap N \beta=\mathscr{V}_{\sigma} \cap N \tau$ and hence, by Lemma 3.4, either $\mathscr{V}_{\alpha} \cap N \beta=0$ or $\mathscr{V} \alpha \subseteq N \beta$. In the first case, $\mathscr{V} \alpha \beta=\mathscr{V} \beta$ and $N \alpha \beta=N \alpha$ while in the second case $\alpha \beta=0$. Hence $\mathscr{F}(H)$ is a semigroup; it is clearly homogeneous.

Theorem 3.7 gives the structure of $\mathscr{F}(H)$ for a large class of semigroups. To state the theorem, we'require the following definition. The biannihilator of a semigroup $S=S^{0}$ is the subset $B(S)=\{x \in S: S x S=0\} ; B(S)$ is clearly an ideal of $S$.

Proposition 3.6. Let $S=S^{0}$ be categorical at zero and let $B(S)$ be the biannihilator of $S$. Then $S=\bar{S} \cup B(S)$ where $\bar{S} \cap B(S)=0$ and $\bar{S}=\bar{S}^{0}$ is a subsemigroup of $S$.

Proof. Let $\bar{S}=(S \backslash B(S)) \cup 0$ and let $a, b \in \bar{S}$ with $a b \neq 0$. Since $a, b \in S \backslash B(S)$ there exist $x, y$ such that $x a \neq 0 \neq b y$. Because $S$ is categorical at zero this implies $x a b y \neq 0$ so that $a b \in \bar{S}$. Hence $\bar{S}$ is a subsemigroup and clearly $S=\bar{S} \cup B(S)$ and $B(S) \cap \bar{S}=0$.

In Proposition 3.6, $S$ is the 0-disjoint union of the subsemigroups $\bar{S}$ and $B(S)$. In general, it is not the 0-direct union of these subsemigroups; see the example after Theorem 3.7.

THEOREM 3.7. Let $H=H^{0}$ be a homogeneous subsemigroup of $\mathscr{L} \mathscr{T}(\mathscr{V})$. Then $\mathscr{F}(H)$ is completely 0-simple if and only if $B(H)=0$.

Proof. Suppose that $\mathscr{F}(H)$ is completely 0 -simple and let $0 \neq \delta \in H$. Then, by Lemma 1.1, there exist $\alpha, \beta \in \mathscr{F}(H)$ such that $\alpha \delta \beta \neq 0$. By the definition of $\mathscr{F}(H)$, there exist $\sigma, \tau \in H$ such that $\mathscr{V} \alpha=\mathscr{V} \sigma, N \beta=N \tau$. If $\sigma \delta=0$ then $0=\mathscr{V} \sigma \delta=\mathscr{V} \alpha \delta$ which implies $\alpha \delta=0$; a contradiction. If $\delta \tau=0$, then $\mathscr{V} \delta \subseteq N \tau=N \beta$ so $\delta \beta=0$; again a contradiction. Hence, since $H$ is categorical at zero, $\sigma \delta \tau \neq 0$. Thus $B(H)=0$.

Conversely, by Lemmas $1.1,3.1$ and 3.2 Corollary, we need only show that $B(H)=0$ implies $\mathscr{F}(H)$ is indecomposable at zero. Let $\alpha, \beta \in \mathscr{F}(H) \mid 0$; then there exist $\sigma, \tau \in H$ such that $\mathscr{V}_{\alpha}=\mathscr{V}_{\sigma}, N \beta=N \tau$. Since $B(H)=0$, there exist $\mu, \nu \in H$ such that $\sigma \mu \neq 0, \nu \tau \neq 0$; thus such that $\mathscr{V} \sigma \cap N \mu=0, \mathscr{V} \nu \cap N \tau=0$. But $H$ homogeneous implies $\operatorname{dim} \mathscr{V} \sigma+\operatorname{dim} N \mu=\operatorname{dim} \mathscr{V}$ and hence $\mathscr{V}=\mathscr{V} \sigma \oplus N \mu$. Let $\gamma^{\prime}$ be a 
nonsingular linear transformation of $\mathscr{V} \sigma$ onto $\mathscr{V} \nu$ and extend $\gamma^{\prime}$ to $\mathscr{V}$ by defining $\mathbf{x} \gamma^{\prime}=0$ for each $\mathbf{x} \in N \mu$; call this linear transformation $\gamma$. Then $N \gamma=N \mu, \mathscr{V} \gamma=\mathscr{V} \nu$ so that $\gamma \in \mathscr{F}(H)$ while $\mathscr{V} \alpha \gamma=\mathscr{V} \sigma \gamma=\mathscr{V} \nu$ and $\mathscr{V} \gamma \cap N \beta=\mathscr{V} \nu \cap N \tau=0$. Thus $\alpha \gamma \neq 0, \gamma \beta \neq 0$ and hence, since $H$ is categorical at zero, $\alpha \gamma \beta \neq 0$.

COROllary. Let $H=H^{0}$ be a homogeneous semigroup of matrices. If $H$ has no nonzero nilpotent ideals then $\mathscr{F}(H)$ is completely 0 -simple; in particular, this is true if $H$ is indecomposable at zero.

Proof. For any semigroup $H=H^{0}, B(H)^{3} \subseteq H B(H) H=0$. Hence, if $H$ has no nonzero nilpotent ideals, $B(H)=0$.

By means of Zorn's Lemma, one can easily show that any homogeneous subsemigroup of $\mathscr{L} \mathscr{T}(\mathscr{V})$ is contained in a maximal such subsemigroup. The following example shows that the maximal homogeneous subsemigroups need not be completely 0-simple.

EXAmple. Let $\Phi$ be a field and let $\mathscr{V}$ be a vector space of dimension 5 over $\Phi$. Let $\mathscr{U}$ be a three dimensional subspace of $\mathscr{V}$ and let

$H=\{\alpha \in \mathscr{L} \mathscr{T}(\mathscr{V}):|\alpha|=2, N \alpha=\mathscr{U}$ and either $\mathscr{V} \alpha \subseteq \mathscr{U}$ or $\mathscr{V} \alpha \cap \mathscr{U}=0\} \cup\{0\}$.

Then $H=H^{0}$ is a maximal homogeneous subsemigroup of $\mathscr{L} \mathscr{T}(\mathscr{V})$. But $B(H)$ $=\{\alpha: \mathscr{V} \alpha \subseteq \mathscr{U}\}$ is nonzero so that $H$ is not completely 0 -simple. In fact, $H=B(H)$ $\cup\left\{\alpha: \mathscr{V}_{\alpha} \cap \mathscr{U}=0\right\}$ where the latter is completely 0 -simple. Further, no element of $H \backslash B(H)$ is a left zero divisor in $H$. Hence, in the notation of Proposition 3.6, $H$ is not the 0 -direct union of $B(H)$ and $\bar{H}$.

Examples can also be given to show that a maximal homogeneous subsemigroup of $\mathscr{L} \mathscr{T}(\mathscr{V})$ can be completely 0 -simple without being irreducible.

For use in later sections, we now consider some properties of the fillout of a semigroup which is indecomposable at zero.

LEMMA 3.8. Let $H=H^{0}$ be a homogeneous subsemigroup of $\mathscr{L} \mathscr{T}(\mathscr{V})$ which is indecomposable at zero and let I be a nonzero ideal of $H$. Then, for each $\alpha \in \mathscr{F}(H)$, there exists $\gamma \in I$ such that $\mathscr{V}_{\alpha}=\mathscr{V}_{\gamma}, N \alpha=N \gamma$.

Proof. From the definition of $\mathscr{F}(H)$, there exist $\sigma, \tau \in H$ such that $N \alpha=N \sigma$ and $\mathscr{V}_{\alpha}=\mathscr{V} \tau$. Since $H$ is indecomposable at zero, there exists $\delta \in I$ such that $\sigma \delta \tau \neq 0$. Let $\gamma=\sigma \delta \tau$ then, since $H$ is homogeneous, $N \gamma=N \sigma=N \alpha, \mathscr{V} \gamma=\mathscr{V} \tau=\mathscr{V} \alpha$ and $\gamma \in I$.

COROLlARY. Let $H=H^{0}$ be indecomposable at zero and let $I$ be a nonzero ideal of H. Then $\mathscr{F}(I)=\mathscr{F}(H)$.

Proof. Since $I \subseteq H$, it is clear that $\mathscr{F}(I) \subseteq \mathscr{F}(H)$. Conversely, by the lemma, if $\alpha \in \mathscr{F}(H)$ there exists $\gamma \in I$ such that $\mathscr{V} \alpha=\mathscr{V} \gamma, N \alpha=N \gamma$. Thus $\alpha \in \mathscr{F}(I)$ and hence $\mathscr{F}(I)=\mathscr{F}(H)$.

It follows from Lemma 3.8 that, if $H$ is indecomposable at zero, $\mathscr{F}(H)$ is obtained from $H$ simply by filling out those $\mathscr{H}$-classes of $\mathscr{L} \mathscr{T}(\mathscr{V})$ which contain members of $H$. The next lemma shows that these are in fact $\mathscr{H}$-classes of $\mathscr{F}(H)$. 
Lemma 3.9. Suppose that $B(H)=0$. Then $\alpha$ and $\beta$ are $\mathscr{H}$-equivalent in $\mathscr{F}(H)$ if and only if $\mathscr{V} \alpha=\mathscr{V} \beta$ and $N \alpha=N \beta$.

Proof. If $\alpha$ and $\beta$ are $\mathscr{H}$-equivalent in $\mathscr{F}(H)$, they certainly have this property in $\mathscr{L} \mathscr{T}(\mathscr{V})$; hence, see [3, p. 57], $\mathscr{V} \alpha=\mathscr{V} \beta$ and $N \alpha=N \beta$. Conversely, we shall show that, if $\mathscr{V} \alpha=\mathscr{V} \beta$, then $\alpha=\gamma \beta$ for some $\gamma \in \mathscr{F}(H)$; the other verifications are similar.

Choose a basis $e_{r+1}, \ldots, e_{n}$ for $N \alpha$ and extend it to a basis $e_{1}, \ldots, e_{n}$ for $\mathscr{V}$. Then $e_{1} \alpha, \ldots, e_{r} \alpha$ are linearly independent. Since $B(H)=0$, there exists $\eta \in H$ such that $\eta \beta \neq 0$; that is, such that range $\eta \cap$ null space $\beta=0$. Let $f_{1}, \ldots, f_{r} \in$ range $\eta$ be such that $\boldsymbol{f}_{i} \beta=e_{i} \alpha(1 \leqq i \leqq r)$ and define $\gamma$ by

$$
\begin{aligned}
\boldsymbol{e}_{i} \gamma & =\boldsymbol{f}_{i} \quad \text { for } 1 \leqq i \leqq r, \\
& =0 \quad \text { for } r<i \leqq n .
\end{aligned}
$$

Then $e_{i} \gamma \beta=e_{i} \alpha(1 \leqq i \leqq n)$ so that $\alpha=\gamma \beta$ and, since $N \gamma=N \alpha$ and $\mathscr{V} \gamma=\mathscr{V} \eta, \gamma \in \mathscr{F}(H)$.

Corollary 1. Let $H=H^{0}$ be a homogeneous subsemigroup of $\mathscr{L} \mathscr{T}(\mathscr{V})$, of rank $r>0$, and suppose that $B(H)=0$. Then each group $\mathscr{H}$-class of $\mathscr{F}(H)$ (i.e. each nonzero maximal subgroup) is isomorphic to the group of all nonsingular linear transformations of rank $r$ over $\Phi$ :

Proof. This is immediate from the lemma and [3, p. 57, Exercise 6(f)].

Corollary 2. Let $H=H^{0}$ be a homogeneous subsemigroup of $\mathscr{L} \mathscr{T}(\mathscr{V})$ which is indecomposable at zero and let I be a nonzero ideal of $H$. Then each element $\alpha$ of $\mathscr{F}(H)$ can be written in the form $\alpha=\gamma \beta \delta$ where $\gamma, \delta \in I$ and $\beta \in \mathscr{F}(H)$.

By the corollary to Theorem $3.7, \mathscr{F}(H)$ is completely 0 -simple and hence there exist idempotents $\zeta$ and $\eta$ such that $\alpha=\zeta \alpha \eta$. By Lemmas 3.8, 3.9, there exist $\gamma, \delta \in I$ such that $(\zeta, \gamma) \cup(\eta, \delta) \in \mathscr{H}$. Then $\gamma \gamma^{-1}=\zeta, \delta^{-1} \delta=\eta$. Thus, if $\beta=\gamma^{-1} \alpha \delta^{-1}, \beta \in \mathscr{F}(H)$ and $\alpha=\gamma \beta \delta$.

LEMmA 3.10. Let $H=H^{0}$ be a homogeneous subsemigroup of $\mathscr{L} \mathscr{T}(\mathscr{V})$ and let $C$ be a nonsingular linear transformation of $\mathscr{V}$. Then $\mathscr{F}\left(C^{-1} H C\right)=C^{-1} \mathscr{F}(H) C$.

Proof. The proof is trivial and is omitted.

Let $S=S^{0}$ be a subsemigroup of $\mathscr{L} \mathscr{T}(\mathscr{V})$. Then we shall call $S$ basic if the inclusion map of $S$ into $\mathscr{L} \mathscr{T}(\mathscr{V})$ is a basic representation of $S$. Thus a representation $\Gamma$ of a semigroup $T=T^{0}$ is basic if and only if $\Gamma(T)$ is basic.

THEOREM 3.11. Let $H=H^{0}$ be a homogeneous subsemigroup of $\mathscr{L} \mathscr{T}(\mathscr{V})$ and suppose that $H$ is indecomposable at zero. Then the following conditions on $H$ are equivalent.

(a) $H$ is basic;

(b) (i) for each $e \in \mathscr{V} \backslash 0$, there exists $\alpha \in H$ such that $e \alpha \neq 0$;

(ii) $[\mathscr{V} H]=\mathscr{V}$;

(c) $\mathscr{F}(H)$ is irreducible;

(d) $I_{n} \in[\mathscr{F}(H)]$. 
Proof. That (a) implies (b) is clear. Suppose that (b) holds and let $\mathscr{Y}$ be a proper subspace of $\mathscr{V}$. Then, by (ii), there exists $f \in \mathscr{V}, \alpha \in H$ such that $f \alpha \notin \mathscr{Y}$. By (i), there exists $g \in \mathscr{Y}, \beta \in H$ such that $g \beta \neq 0$. Let $e_{r+1}, \ldots, e_{n}$ be a basis for $N \beta$ and extend $g=e_{r}, e_{r+1}, \ldots, e_{n}$ to a basis $e_{1}, \ldots, e_{n}$ for $\mathscr{V}$ and let $f_{1}, \ldots, f_{r}$ be a basis for $\mathscr{V}_{\alpha}$ where $f_{r}=f \alpha$. Define $\gamma \in \mathscr{L} \mathscr{T}(\mathscr{V})$ as follows:

$$
\begin{aligned}
\boldsymbol{e}_{i} \gamma & =\boldsymbol{f}_{i} & & 1 \leqq i \leqq r, \\
& =0 & & r<i \leqq n .
\end{aligned}
$$

Then $N \gamma=N \beta, \mathscr{V} \gamma=\mathscr{V}_{\alpha}$ so that $\gamma \in \mathscr{F}(H)$. However $\mathscr{Y} \gamma \nsubseteq \mathscr{Y}$ since $e_{r} \in \mathscr{Y}$ but $\boldsymbol{e}_{r} \gamma=\boldsymbol{f}_{r}=f \alpha \notin \mathscr{Y}$. Hence $\mathscr{F}(H)$ is irreducible.

That (c) implies (d) is immediate from [3, Lemma 5.32], so suppose that (d) holds. By Lemma 3.8, if $I$ is a nonzero ideal of $H$, then $\mathscr{F}(I)=\mathscr{F}(H)$ and hence $I_{n} \in[\mathscr{F}(I)]$. Suppose $\mathscr{U} I=0$; then $\mathscr{U} \subseteq N \alpha$ for each $\alpha \in I$ and hence, from the definition of $\mathscr{F}(I), \mathscr{U} \subseteq N \beta$ for each $\beta \in \mathscr{F}(I)$. But this implies $\mathscr{U}[\mathscr{F}(I)]=0$ which, since $I_{n} \in[\mathscr{F}(I)]$ requires $\mathscr{U}=0$. Suppose that $\mathscr{V} I \subseteq \mathscr{U}$ where $\mathscr{U}$ is a subspace of $\mathscr{V}$. Then, from the definition of $\mathscr{F}(I)$, it follows that $\mathscr{V} \mathscr{F}(I) \subseteq \mathscr{U}$ and hence $[\mathscr{V} \mathscr{F}(I)]$ $\subseteq \mathscr{U}$. Since $[\mathscr{V} \mathscr{F}(I)]=[\mathscr{V}[\mathscr{F}(I)]]$, this however implies $\mathscr{V} I_{n} \subseteq \mathscr{U}$ so that $\mathscr{U}=\mathscr{V}$. Hence $H$ is basic.

The final theorem of this section shows that, for completely 0 -simple semigroups, basic representations in our sense are the same as the basic representations considered by Clifford in [1], [2].

THEOREM 3.12. Let $S=\mathscr{M}^{0}(G ; I, \Lambda ; P)$ be a completely 0 -simple semigroup and let $\Gamma$ be a representation of $S$ of degree $n$ over a field $\Phi$. Then the following conditions on $\Gamma$ are equivalent.

(a) $\Gamma$ is basic;

(b) $\Gamma$ is basic in the sense of Clifford.

Proof. Suppose that $\Gamma$ is not basic in the sense of Clifford. Then it is immediate from [3, Theorem 5.48] that there exist subspaces $\mathscr{U}$ and $\mathscr{Y}$, not both trivial, such that $\mathscr{U} \Gamma(S)=0, \mathscr{V} \Gamma(S) \subseteq \mathscr{Y}$, where $\mathscr{V}$ is the space of all $1 \times n$ row matrices over $\Phi$. Hence $\Gamma$ is not basic.

Conversely, suppose $\Gamma$ is basic in the sense of Clifford. Then, without loss of generality, we may assume that, for each $(a ; i, \lambda) \in S$,

$$
\Gamma(a ; i, \lambda)=\left[\begin{array}{ll}
\Gamma^{0}\left(p_{1 i} a p_{\lambda 1}\right) & \Gamma^{0}\left(p_{1 i} a\right) Q_{\lambda} \\
R_{i} \Gamma^{0}\left(a p_{\lambda 1}\right) & R_{i} \Gamma^{0}(a) Q_{\lambda}
\end{array}\right]
$$

where $\Gamma^{0}$ is a representation of $G \cup 0$ of degree $r$ over $\Phi$. Then $n=r+\operatorname{rank} \Omega$, where $\Omega$ is the extending matrix of $\Gamma$.

Let $\mathscr{G}$ denote the group of all nonsingular linear transformations of degree $r$ over $\Phi$, and let $C=\mathscr{M}^{0}(\mathscr{G} ; I, \Lambda ; \bar{P})$ where $\bar{P}=\left[\Gamma^{0}\left(p_{\lambda_{i}}\right)\right]$. Then the mapping $\Delta$ defined by

$$
\Delta(X ; i, \lambda)=\left[\begin{array}{cc}
\Gamma^{\circ}\left(p_{1 i}\right) X \Gamma^{\circ}\left(p_{\lambda 1}\right) & \Gamma^{\circ}\left(p_{1 i}\right) X Q_{\lambda} \\
R_{i} X \Gamma^{\circ}\left(p_{\lambda 1}\right) & R_{i} X Q_{\lambda}
\end{array}\right]
$$


is a representation of $C$ of degree $n$ over $\Phi$. Since $\Gamma$ is basic in the sense of Clifford, so is $\Delta$ and, since $\Delta$ extends the faithful representation of $\mathscr{G}$, which is irreducible, it follows from [3, Theorem 5.51], that $\Delta$ is irreducible.

Let $\varphi$ denote the mapping of $S$ into $C$ defined by $(x ; i, \lambda) \varphi=\left(\Gamma^{0}(x) ; i, \lambda\right)$. Then it is easy to see that $\varphi$ is a 0 -restricted homomorphism of $S$ into $C$ such that, for each $(x ; i, \lambda) \in S, \Gamma(x ; i, \lambda)=\Delta[(x ; i, \lambda) \varphi]$. Further, it is clear that each $\mathscr{H}$-class of $C$ contains an element of $S \varphi$; thus each $\mathscr{H}$-class of $\Delta(C)$ contains an element of $\Gamma(S)$. By Lemma 3.8, this implies $\Delta(C) \subseteq \mathscr{F}(\Gamma(S))$ and so, since $\Delta(C)$ is irreducible, $\mathscr{F}(\Gamma(S))$ is irreducible. Hence, by Theorem $3.11, \Gamma(S)$ is a basic subsemigroup of $[\Phi]_{n}$ so that $\Gamma$ is basic.

By [3, Lemma 5.32], if $\Gamma$ is an irreducible representation of a completely 0 -simple semigroup, then $[\Gamma(S)]$ contains the identity matrix. Clifford has given an example [3, p. 192, Exercise 8], to show that a basic representation of a completely 0 -simple semigroup can have a null irreducible constituent. If $\Gamma$ is such a representation of $S$ then, clearly, $[\Gamma(S)]$ cannot contain the identity matrix although, by Lemma 3.9, Corollary 2 and Theorem 3.11, the algebra $[\Gamma(S) \mathscr{F}[\Gamma(S)] \Gamma(S)]$ does.

4. Homogeneous representations. Let $S=S^{0}$ be a semigroup and let $\Gamma$ be a representation of $S$ of degree $n$ over a field $\Phi$. Then $\Gamma$ is said to be homogeneous if $\Gamma(S)$ is a homogeneous subsemigroup of $[\Phi]_{n}$. Thus $\Gamma$ is homogeneous if and only if $S=M(\Gamma)$, where $M(\Gamma)$ is defined as in $\S 2$.

Let $S=S^{0}$ be a semigroup without nonzero nilpotent ideals and let $\Gamma$ be a 0 -restricted homogeneous representation of $S$ of degree $n$ over a field $\Phi$. Then, since $\Gamma$ is 0 -restricted, it follows from the Corollary to Lemma 3.1 that $S$ is categorical at zero. Further, by the corollary to Theorem 3.7, $\Gamma$ is a 0 -restricted homomorphism of $S$ into the completely 0 -simple semigroup $\mathscr{F}(\Gamma(S))$. It therefore follows from Theorem 1.9 that $\Gamma$ induces a (unique) representation of $\mathscr{P}(S)$ such that the diagram

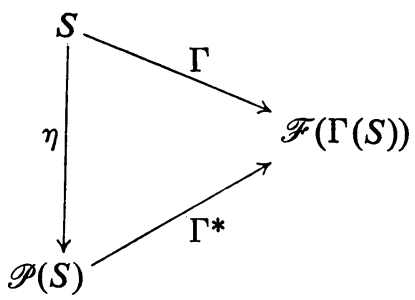

commutes; recall that $\mathscr{P}(S)$ is the coreflection of $S$ in the category of $p$-regular semigroups and 0-restricted homomorphisms. We call $\Gamma^{*}$ the representation induced by $\Gamma$ and $\Gamma$ the restriction of $\Gamma^{*}$ to $S$.

Let $S=S^{0}$ be the 0 -direct union of a family $\left\{S_{i}: i \in I\right\}$ of 0 -primary semigroups and let $\Gamma$ be a homogeneous 0 -restricted representation of $S$ of degree $n$ over $\Phi$. Then, in general, we cannot say much about the representation $\Gamma^{*}$ of $\mathscr{P}(S)$ induced 
by $\Gamma$. If, however, $\Gamma \mid S_{i}$ is basic, for each $i \in I$, then we can give the structure of $\Gamma^{*}$ modulo representations of completely 0 -simple semigroups.

THEOREM 4.1. Let $S=S^{0}$ be the 0-direct union of a family $\left\{S_{i}: i \in I\right\}$ of 0 -primary semigroups. Let $\Gamma$ be a proper 0 -restricted homogeneous representation of $S$ of degree $n$ over a field $\Phi$. Suppose that, for each $i \in I, \Gamma \mid S_{i}$ decomposes into a null representation and a basic representation. Then $I$ is finite, $I=\{1,2, \ldots, r\}$, with $r \leqq n$, and $\Gamma$ is equivalent to a representation of the form

$$
\left[\begin{array}{lll}
\Gamma_{1} & & 0 \\
& \ddots & \\
0 & & \Gamma_{r}
\end{array}\right]
$$

where, for each $i \in I, \Gamma_{i}(x)=0$ if $x \notin S_{i}$ and $\Gamma_{i} \mid S_{i}$ is a homogeneous 0 -restricted basic representation of $S_{i}$, equivalent to the proper part of $\Gamma \mid S_{i}$.

Proof. Pick $! \in I$ and rearrange the basis for the underlying vector space to obtain an equivalent representation $\Delta$ of $S$ so that, for each $x \in S_{1}$,

$$
\Delta(x)=\left[\begin{array}{cc}
\Gamma_{1}(x) & 0 \\
0 & 0
\end{array}\right]
$$

where $x \rightarrow \Gamma_{1}(x)$ is a proper 0 -restricted representation of $S_{1}$ of degree $r_{1}$ over $\Phi$. By hypothesis, $\Gamma_{1}$ is basic and hence, by Theorem 3.11, $I_{r_{1}} \in\left[\mathscr{F}\left(\Gamma_{1}\left(S_{1}\right)\right)\right]$. Thus, by Lemma 3.9, Corollary 2, there exist finite sets of elements $u_{j}, v_{j} \in S_{1}$ and $X_{j} \in[\Phi]_{r_{1}}$ $(1 \leqq j \leqq s)$ such that

$$
E=\left[\begin{array}{cc}
I_{r_{1}} & 0 \\
0 & 0
\end{array}\right]=\sum_{1}^{s}\left[\begin{array}{cc}
\Gamma_{1}\left(u_{j}\right) X_{j} \Gamma_{1}\left(v_{j}\right) & 0 \\
0 & 0
\end{array}\right] .
$$

For each $x \in S_{k}, k \neq 1, \Gamma(x) \Gamma\left(S_{1}\right)=0=\Gamma\left(S_{1}\right) \Gamma(x)$. Hence $\Delta(x) E=0=E \Delta(x)$. Let $\Delta(x)$ have the form

$$
\left[\begin{array}{ll}
A(x) & B(x) \\
C(x) & D(x)
\end{array}\right]
$$

where $A(x)$ is $r_{1} \times r_{1}$ and $x \notin S_{1}$. Then, multiplying on left and right by $E$, we find $A(x)=0, \mathrm{~g}(x)=0, C(x)=0$. Hence $\Delta$ decomposes into

$$
\left[\begin{array}{cc}
\Gamma_{1} & 0 \\
0 & \Delta_{1}
\end{array}\right]
$$

where $\Gamma_{1}(x)=0$ if $x \notin S_{1}$ and $\Delta_{1}\left(S_{1}\right)=0$. Since $\Gamma$ is assumed to be proper, $\Delta_{1}$ is a proper homogeneous 0 -restricted representation of $0\left\{S_{i}: i \in I, i \neq 1\right\}$. Hence, since for each $i, \Delta \mid S_{i}$ is equivalent to $\Gamma \mid S_{i}$, the hypothesis of the theorem holds for $\Delta_{1}$ and $0\left\{S_{i}: i \in I, i \neq 1\right\}$. Thus, repeating the process at most $n$ times, we obtain the result. 
Corollary. Let $S=0\left\{S_{i}: i \in I\right\}$ be as in the statement of the theorem and suppose that, for each $i \in I$, each proper 0-restricted homogeneous representation of $S_{i}$ is basic. Then every proper 0-restricted homogeneous representation of $S$ is equivalent to one of the form (4.1).

It has been pointed out that any 0 -restricted homogeneous representation $\Gamma$, of a semigroup $S=S^{0}$, which is categorical at zero and without nonzero nilpotent ideals, induces a (unique) 0 -restricted homogeneous representation $\Gamma^{*}$ of $\mathscr{P}(S)$ of the same degree. The next three theorems show that the correspondence preserves equivalence, decomposition and reduction. (That this is the case, when $\mathscr{P}(S)$ is a homomorphic image of $S$, is obvious.)

THEOREM 4.2. Let $S=S^{0}$ be the 0-direct union of a family $\left\{S_{i}: i \in I\right\}$ of 0-primary semigroups. Let $\Gamma, \Delta$ be 0-restricted homogeneous representations of $S$ of degree $n$ over a field $\Phi$. Then $\Gamma$ is equivalent to $\Delta$ if and only if the representation $\Gamma^{*}$ of $\mathscr{P}(S)$ induced by $\Gamma$ is equivalent to the representation $\Delta^{*}$ induced by $\Delta$.

Proof. Clearly the equivalence of $\Gamma^{*}$ and $\Delta^{*}$ implies that of $\Gamma$ and $\Delta$. Conversely, suppose that there exists a nonsingular $n \times n$ matrix $C$ such that, for each $x \in S$, $\Delta(x)=C^{-1} \Gamma(x) C$. Then the mapping $x \rightarrow C^{-1} \Gamma^{*}(x) C$ is a 0 -restricted homomorphism of $\mathscr{P}(S)$ into $[\Phi]_{n}$; in fact, it is into $C^{-1} \mathscr{F}(\Gamma(S)) C$ since $\Gamma^{*}(\mathscr{P}(S)) \subseteq \mathscr{F}(\Gamma(S))$. By Lemma $3.10, C^{-1} \mathscr{F}(\Gamma(S)) C=\mathscr{F}\left(C^{-1} \Gamma(S) C\right)$ and the latter is just $\mathscr{F}(\Delta(S))$. Thus $\Delta^{*}$ and $x \rightarrow C^{-1} \Gamma^{*}(x) C$ both extend $\Delta$ to homomorphisms of $\mathscr{P}(S)$ into the completely 0 -simple semigroup $\mathscr{F}(\Delta(S))$. Hence by the uniqueness property of Theorem 1.9, the two mappings are identical and $\Gamma^{*}$ and $\Delta^{*}$ are equivalent.

THEOREM 4.3. Let $S=S^{0}$ be the 0-direct union of a family $\left\{S_{i}: i \in I\right\}$ of 0-primary semigroups. Let $\Gamma$ be a 0 -restricted homogeneous representation of $S$ of degree $n$ over a field $\Phi$. Then $\Gamma$ decomposes into the direct sum of representations $\Gamma_{1}$ and $\Gamma_{2}$ of degrees $n_{1}$ and $n_{2}$ if and only if the same is true of $\Gamma^{*}$.

Proof. If $\Gamma^{*}$ decomposes into the direct sum of two representations then clearly so does $\Gamma$. Suppose conversely that, for each $s \in S$,

$$
\Gamma(s)=\left[\begin{array}{cc}
\Gamma_{1}(s) & 0 \\
0 & \Gamma_{2}(s)
\end{array}\right]
$$

Then, for each $x \in S \eta$,

$$
\Gamma^{*}(x)=\left[\begin{array}{cc}
\Gamma_{1}(s) & 0 \\
0 & \Gamma_{2}(s)
\end{array}\right]
$$

where $x=s \eta$. It thus suffices to show that, if $T=0\left\{T_{i}: i \in I\right\}$ is a homogeneous semigroup of matrices of the form (4.2), where each $T_{i}$ is 0 -primary, the $p$-regular semigroup of matrices generated by $T$ consists of matrices of the form (4.2). This is however an immediate consequence of Theorem 1.9(c) and Lemma 3.3. 
COROllaRY 1. Let $S=S^{0}$ be a 0 -primary semigroup and let $\Gamma$ be a proper 0 -restricted homogeneous representation of $S$ of degree $n$ over a field $\Phi$. Then there is a one-to-one correspondence between the indecomposable constituents of $\Gamma$ and the indecomposable constituents of the representation $\Gamma^{*}$ of $\mathscr{C}(S)$ induced by $\Gamma$. Each indecomposable constituent of $\Gamma$ is homogeneous and 0-restricted.

Proof. That there is a one-to-one correspondence between the indecomposable constituents of $\Gamma$ and those of $\Gamma^{*}$ is immediate from the theorem. Since each representation of a completely 0 -simple semigroup is homogeneous and 0-restricted, the latter result in the statement of the theorem follows from the former.

If $S$ and $\Gamma$ are as in Theorem 4.3 then we know that each indecomposable constituent of $\Gamma$ is the restriction to $S$ of an indecomposable constituent of $\Gamma^{*}$. In general, we do not know that these are homogeneous; however, if $\Gamma$ satisfies the conditions of Theorem 4.1, we can apply Corollary 1 to obtain the following result.

COROLlary 2. Let $S$ and $\Gamma$ be as in Theorem 4.1, then each indecomposable constituent of $\Gamma$ is basic and homogeneous.

Let $S=S^{0}$ be a semigroup and let $\Gamma$ be an irreducible representation of $S$ of degree $n$ over a field $\Phi$. Then, by [6, Lemma 5], $S$ is indecomposable at zero. Hence there is no loss of generality in Theorem 4.5 below.

LEMMA 4.4. Let $\Phi$ be a field and let $H$ be a 0 -primary homogeneous subsemigroup of $[\Phi]_{n}$. Suppose that each element of $H$ has the form

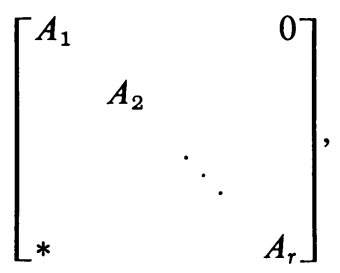

where each $A_{i}$ is $n_{i} \times n_{i}$. Then the subsemigroup $C$ of $\mathscr{F}(H)$ generated by $H$ consists of elements of the form (4.3).

Proof. $C=\bigcup\left\{H_{n}: n \geqq 0\right\}$ where $H_{0}=H$ and $H_{n+1}$ is generated by $H_{n}$ and the inverses of elements of $H_{n}$ whose square is nonzero. Suppose that each element of $H_{n}$ has the form (4.3). Then it follows from Lemma 3.3, by an easy induction on $r$, that the inverse of any element of $H_{n}$ also has the form (4.3). Hence, by the block multiplication rule, each element of $H_{n+1}$ has this form and, by induction, the same is true of each element of $C$.

THEOREM 4.5. Let $S=S^{0}$ be a 0-primary semigroup and let $\Gamma$ be a homogeneous 0 -restricted representation of $S$, of degree $n$ over a field $\Phi$. Then the irreducible constituents of $\Gamma$ are the restrictions to $S$ of the irreducible constituents of the representation $\Gamma^{*}$ of $\mathscr{C}(S)$ induced by $\Gamma$. 
Proof. Let $\Gamma$ have ultimately reduced form

$$
\left[\begin{array}{cccc}
\Gamma_{1} & & & 0 \\
& \Gamma_{2} & & \\
& & \ddots & \\
& & & \Gamma_{r}
\end{array}\right] \text {, }
$$

where each $\Gamma_{i}$ is $n_{i} \times n_{i}$. Then each element of $\Gamma^{*}(s \eta)$ has this form and so, by Lemma 4.4, has each element of the completely 0-simple subsemigroup $C$ of $\mathscr{F}(\Gamma(S))$ generated by $\Gamma(S)$. But, by Theorem $1.6, C=\Gamma^{*}(\mathscr{C}(S))$ so that, for each $x \in \mathscr{C}(S)$,

$$
\left[\begin{array}{cccc}
\Gamma_{1}^{*} & & & 0 \\
& \Gamma_{2}^{*} & & \\
& & \ddots & \\
& & & \Gamma_{r}^{*}
\end{array}\right] \text {, }
$$

where each $\Gamma_{i}^{*}$ is $n_{i} \times n_{i}$ and, for each $s \in S, \Gamma_{i}(s)=\Gamma_{i}^{*}(S \eta)$. Now $\Gamma_{i}(S) \subseteq \Gamma_{i}^{*}(\mathscr{C}(S))$ hence the irreducibility of $\Gamma_{i}$ implies that $\Gamma_{i}^{*}$ is also irreducible. Therefore $\Gamma^{*}$ has ultimately reduced form (4.5).

COROLLARY 1. With the hypotheses of Theorem 4.5, each nonnull irreducible constituent of $\Gamma$ is homogeneous and 0-restricted.

COROLlaRY 2. With the hypotheses of Theorem $4.5, \Gamma$ is irreducible if and only if the same is true of $\Gamma^{*}$.

5. 0-simple and 0-bisimple semigroups. Since any 0 -simple semigroup of matrices is a homogeneous semigroup and every nonnull homomorphic image of a 0 -simple semigroup is again 0 -simple, the theory in $\$ 4$ applies, in particular, to 0 -simple semigroups. Specifically, we have Theorem 5.1.

THEOREM 5.1. Let $S=S^{0}$ be a 0 -simple semigroup and let $\Phi$ be a field. Then $S$ is indecomposable at zero and, if $S$ has a nonnull representation over $\Phi$, then $S$ is categorical at zero.

(a) Let $\Gamma$ be a nonnull representation of $S$ of degree $n$ over $\Phi$. Then $\Gamma$ induces a (unique) representation $\Gamma^{*}$ of $\mathscr{C}(S)$ of the same degree over $\Phi$ so that the diagram

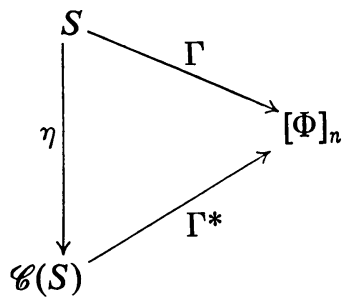

commutes. 
(b) The indecomposable constituents of $\Gamma$ are the restrictions to $S$ of the indecomposable constituents of $\Gamma^{*}$.

(c) The irreducible constituents of $\Gamma$ are the restrictions to $S$ of the irreducible constituents of $\Gamma^{*}$.

(d) Two representations $\Gamma$ and $\Delta$ of $S$, of degree $n$ over $\Phi$, are equivalent if and only if the induced representations $\Gamma^{*}$ and $\Delta^{*}$ of $\mathscr{C}(S)$ are equivalent.

Theorem 5.1 was proved in [6] for the case in which $S=S^{0}$ has a nonzero idempotent. If this occurs, $\mathscr{C}(S)$ is a 0 -restricted homomorphic image of $S$; in fact, it is the maximum weakly 0 -cancellative 0 -restricted homomorphic image of $S$. To conclude this section, we show that if $S=S^{0}$ is 0-bisimple and categorical at zero then $\mathscr{C}(S)$ is also a 0 -restricted homomorphic image of $S$. A consequence of this result and Theorem 5.1 is that any 0-bisimple (bisimple) semigroup of matrices is completely 0 -simple (completely simple). Note however that not every 0 -simple semigroup of matrices is completely 0-simple; see [3, p. 192, Exercise 7(b)].

THEOREM 5.2. Let $S=S^{0}$ be a 0 -bisimple semigroup. Then $S$ is completely 0-simple if and only if it is weakly 0-cancellative and satisfies

$$
\text { if } a x=x \neq 0 \text { or } x a=x \neq 0 \text { then } a^{2}=a .
$$

Proof. It is straightforward to verify that any completely 0-simple semigroup satisfies these conditions. Conversely, suppose that the conditions are satisfied; we show that $S$ has a primitive idempotent.

If $S$ has only one nonzero element $a$ then $a^{2}=a$ so that $a$ is a primitive idempotent. If $|S|>2$, let $a, b$ be distinct nonzero elements of $S$ then there exists $c \in S$ such that $S^{1} a=S^{1} c, c S^{1}=b S^{1}$. Since $a$ and $b$ are distinct, $c$ is not equal to both $a$ and $b$; say $a \neq c$. Then there exist $x, y \in S$ such that $a=x c, c=y a$. Thus $a=x y a \neq 0$ so that $x y$ is a nonzero idempotent of $S$ by $\left({ }^{*}\right)$. Since $S$ is weakly 0 -cancellative, any nonzero idempotent is primitive; in particular, $x y$ is primitive. Hence $S$ is completely 0 -simple.

COROllary 1. Let $S=S^{0}$ be a 0 -bisimple semigroup which is categorical at zero. Then $\mathscr{C}(S)$ is a 0 -restricted homomorphic image of $S$.

Proof. Let $\left\{\sigma_{i}: i \in I\right\}$ be the set of all 0-restricted congruences on $S$ such that $S / \sigma_{i}$ is weakly 0 -cancellative and such that

$$
(a x, x) \in \sigma_{i} \text { or }(x a, x) \in \sigma_{i} \text {, where } x \neq 0 \text {, implies }\left(a^{2}, a\right) \in \sigma_{i} ;
$$

since $S$ is categorical and indecomposable at zero, it follows, from [6, Theorem 1],

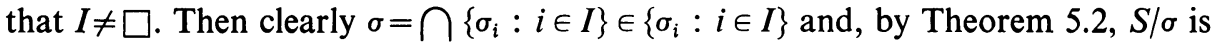
completely 0 -simple.

Since $\mathscr{C}(S)$ is completely 0 -simple any subsemigroup of $\mathscr{C}(S)$ is weakly 0-cancellative and obeys $\left({ }^{*}\right)$; in particular, this is true of $S \eta$. Thus there is a unique 0 -restricted homomorphism $\zeta$ of $S / \sigma$ into $\mathscr{C}(S)$ and a unique 0 -restricted homomorphism $\xi$ of $\mathscr{C}(S)$ into $S / \sigma$ so that the diagram 


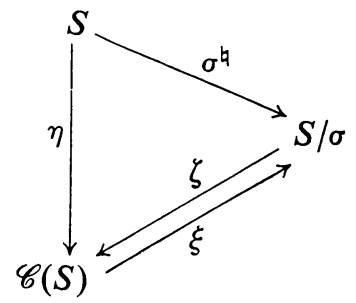

commutes. Hence, by the uniqueness of $\zeta$ and $\xi$, we have $S / \sigma \cong \mathscr{C}(S)$.

COROLlaRY 2. Let $H=H^{0}$ be a homogeneous subsemigroup of $\mathscr{L} \mathscr{T}(\mathscr{V})$. If $H$ is 0-bisimple then $H$ is completely 0 -simple.

Proof. From Theorem 3.7, $\mathscr{F}(H)$ is completely 0 -simple and hence each subsemigroup of $\mathscr{F}(H)$ is weakly 0 -cancellative and obeys $\left(^{*}\right)$; in particular $H$ obeys these conditions. Thus, by Theorem $5.2, H$ is completely 0 -simple.

6. The structure of basic and irreducible representations. The main theorem of this section (Theorem 6.2) gives a construction for all basic representations of an arbitrary semigroup in terms of basic representations of completely 0-simple semigroups. Every irreducible representation of a semigroup is basic so that Theorem 6.2 applies here, but in this case the construction can be simplified.

Recall that a pair $(U, N)$ of ideals of a semigroup $S=S^{0}$ is called primary if

(a) $S / U$ is indecomposable at zero;

(b) $N / U$ is categorical at zero.

We define the pair $(U, N)$ of ideals of $S$ to be secondary if $N / U$ is 0 -primary; thus every primary pair is secondary. Corollary 1 to the following lemma shows that associated with any secondary pair of ideals of $S$ there is a primary pair of ideals of $S$.

LEMMA 6.1. Let $S=S^{0}$ be a semigroup and let $U, N$ be ideals of $S$ such that $U \subset N$ and $N / U$ is indecomposable at zero. Then $N . \cdot U=\{x \in S: N x \subseteq U\}$ is the unique ideal $\bar{U}$ of $S$ such that $S / \bar{U}$ is indecomposable at zero and $\bar{U} \cap N=U$.

Proof. Let $x \in N \cdot{ }^{\cdot} U, s, t \in S^{1}$; then $N s x t \subseteq N x t \subseteq U t \subseteq U$ since $U, N$ are ideals of $S$. Hence $N \cdot \cdot U$ is an ideal of $S$ and, since $N / U$ is indecomposable at zero, $N \cdot \cdot U \cap N=U$.

Let $a, b \in S \backslash N$. $\cdot U$; then there exist $m, n \in N$ such that $m a \notin U, n b \notin U$. Since $N$ is an ideal of $S$ and $N / U$ is indecomposable at zero, this implies $\operatorname{maNnb} \notin$. Thus $a N b \nsubseteq U . \cdot N$ and so $S / N$. $^{\cdot} U$ is indecomposable at zero.

Suppose that $\bar{U}$ is an ideal of $S$ such that $S / \bar{U}$ is indecomposable at zero and $\bar{U} \cap N=U$. If $a \notin \bar{U}$ then there exists $n \in N$ such that $n a \notin U=N \cap \bar{U}$. Thus $a \notin N$. $U$. If $a \notin N . \cdot U$ then there exists $n \in N$ such that $n a \notin U=N \cap N$. $U$. Thus $a \notin \bar{U}$. Hence $\bar{U}=N \cdot \cdot U$. 
COROLlary 1. Let $S=S^{0}$ be a semigroup and let $(U, N)$ be a secondary pair of ideals of $S$. Then $(N \cdot \cdot U, N \cup N \cdot \cdot U)$ is the unique primary pair of ideals $(\bar{U}, \bar{N})$ such that the diagram

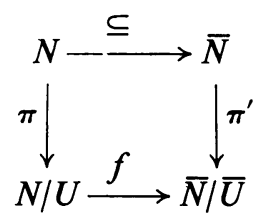

commutes where $\pi$ and $\pi^{\prime}$ are the natural homomorphisms and $f$ is an isomorphism.

Lemma 6.1 also shows that, at least for semisimple semigroups $S=S^{0}$, there is no shortage of ideals $U$ such that $S / U$ is indecomposable at zero.

COROLlARY 2. Let $S=S^{0}$ be a semisimple semigroup and let $a, b \in S$. Then $(a, b) \in \mathscr{J}$ if and only if, for each ideal $U$ of $S$ such that $S / U$ is indecomposable at zero, either both $a, b \in U$ or both $a, b \in S \backslash U$.

THEOREM 6.2. Let $S=S^{0}$ be a semigroup and let $\Phi$ be a field.

(I) Let $\Gamma$ be a basic representation of $S$ of degree $n$ over $\Phi$.

(a) $(V, M)$ is a primary pair of ideals of $S$ and $\Gamma$ induces a unique basic representation $\Gamma^{*}$ of $\mathscr{C}(M / V)$ such that the diagram

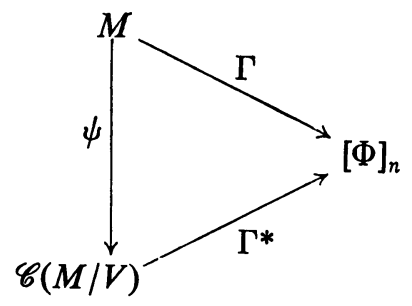

commutes where $\psi=\pi \eta ; \pi$ the natural homomorphism of $M$ onto $M / V$ and $\eta$ the natural homomorphism of $M / V$ into $\mathscr{C}(M / V)$.

(b) There exist finite sets of elements $u_{i}, v_{i} \in M, X_{i} \in \mathscr{F}(\Gamma(M))=\mathscr{F}\left(\Gamma^{*}(\mathscr{C}(M / V))\right)$ such that

(c) For each $s \in S$,

$$
\sum_{i=1}^{r} \Gamma^{*}\left(u_{i} \psi\right) X_{i} \Gamma^{*}\left(v_{i} \psi\right)=I_{n}
$$

$$
\Gamma(s)=\sum_{1}^{r} \Gamma^{*}\left(u_{i} \psi\right) X_{i} \Gamma^{*}\left(v_{i} s \psi\right)
$$

(II) Let $(U, N)$ be a secondary pair of ideals of $S$ and let $\Gamma^{*}$ be a 0 -restricted basic representation of $\mathscr{C}(N / U)$ of degree $n$ over $\Phi$.

(d) There exist $u_{i}, v_{i} \in N, X_{i} \in \mathscr{F}\left(\Gamma^{*}(\mathscr{C}(N / U))\right)$ such that (6.2) holds. For such elements, the mapping $\Gamma: S \rightarrow[\Phi]_{n}$ defined by (6.3) is a basic representation of $S$ with vanishing ideal $N . \cdot U$. Further, the diagram (6.1) commutes with $M$ replaced by $N$ and $V$ replaced by $U$. 
(e) If $\Delta$ is any representation of $S$ of degree $n$ over $\Phi$ such that $\Delta|N=\Gamma| N$ then $\Delta=\Gamma$; in particular, $\Gamma$ is independent of the particular choice of elements satisfying (6.2).

Proof. (I)(a) Let $\mathscr{V}$ be the underlying vector space for $\Gamma$ and let $a, b \in S \backslash V$. Then there exists $\mathbf{v} \in \mathscr{V}$ such that $\mathbf{v} \Gamma(a) \neq 0$. Let $L=S^{1} b S^{1}$; then $L$ is an ideal of $S$ such that $\Gamma(L) \neq 0$ and hence there exists $x \in L$ such that $(v \Gamma(a)) \Gamma(x) \neq 0$. This implies $\Gamma(a x) \neq 0$ and therefore $a x \notin V$. Since $x \in S^{1} b S^{1}, a x \notin V$ implies $a S b \nsubseteq V$ or $a b \notin V$. Similarly the latter implies $a S a b \nsubseteq V$ or $a \cdot a b \notin V$. Thus, in any case, $a S b \nsubseteq V$ and hence $S / V$ is indecomposable at zero. By definition, $\Gamma(M)$ is homogeneous and thus, from Lemma $3.1, M / V$ is categorical at zero. Therefore $(V, M)$ is a primary pair.

By the remarks at the beginning of $\S 4, \Gamma$ thus induces a unique representation $\Gamma^{*}$ of $\mathscr{C}(M / V)$ such that diagram (6.1) commutes. Hence to complete the proof of (a) we need only show that $\Gamma^{*}$ is basic. Since $\Gamma$ is basic and $\Gamma \mid M$ is homogeneous, it follows from Theorem 3.11 that $\Gamma \mid M$ is basic and therefore $\mathscr{F}(\Gamma(M))$ is irreducible. But $\Gamma(M) \subseteq \Gamma^{*}(\mathscr{C}(M / V)) \subseteq \mathscr{F}(\Gamma(M))$; hence $\mathscr{F}(\Gamma(M))=\mathscr{F}\left(\Gamma^{*}(\mathscr{C}(M / V))\right)$. Thus $\mathscr{F}\left(\Gamma^{*}(\mathscr{C}(M / V))\right)$ is irreducible and so, by Theorem 3.11 again, $\Gamma^{*}$ is basic.

(b) By Theorem 3.11 and Corollary 2 to Lemma 3.9, there exist $u_{i}, v_{i} \in M$, $X_{i} \in \mathscr{F}(\Gamma(M))=\mathscr{F}\left(\Gamma^{*}(\mathscr{C}(M / V))\right)$ such that (6.2) holds. Then, for each $s \in S$,

$$
\Gamma(s)=I_{n} \Gamma(s)=\left(\sum_{1}^{r} \Gamma^{*}\left(u_{i} \psi\right) X_{i} \Gamma^{*}\left(v_{i} \psi\right)\right) \Gamma(s)=\sum_{1}^{r}\left\{\Gamma^{*}\left(u_{i} \psi\right) X_{i} \Gamma^{*}\left(v_{i} \psi\right) \Gamma(s)\right\} .
$$

By the commutativity of $(6.1), \Gamma^{*}\left(v_{i} \psi\right) \Gamma(s)=\Gamma\left(v_{i}\right) \Gamma(s)=\Gamma^{*}\left(v_{i} s \psi\right) ;(M$ is an ideal of $S$ so that $\left.v_{i} s \in M\right)$. Hence (6.3) holds and the proof of (I) is complete.

(II)(d). Let $\Gamma^{*}$ be a 0 -restricted basic representation of $\mathscr{C}(N / U)$ of degree $n$ over $\Phi$. Then, since $\mathscr{F}\left(\Gamma^{*}(N \psi)\right)=\mathscr{F}\left(\Gamma^{*}(\mathscr{C}(N / U))\right)$, there exist elements $u_{i}, v_{i} \in N$, $X_{i} \in \mathscr{F}\left(\Gamma^{*}(N \psi)\right), 1 \leqq i \leqq r$, such that (6.2) holds. Let $u_{i}, v_{i} \in N, X_{i} \in \mathscr{F}\left(\Gamma^{*}(N \psi)\right)$, $1 \leqq i \leqq r$, be such a set of elements and define, for $s \in S$,

Then

$$
\Gamma(s)=\sum_{i=1}^{r} \Gamma^{*}\left(u_{i} \psi\right) X_{i} \Gamma^{*}\left(v_{i} s \psi\right)
$$

$$
\begin{aligned}
\Gamma(s)=\Gamma(s) I_{n} & =\sum_{i=1}^{r} \sum_{j=1}^{r} \Gamma^{*}\left(u_{i} \psi\right) X_{i} \Gamma^{*}\left(v_{i} s \psi\right) \Gamma^{*}\left(u_{j} \psi\right) X_{j} \Gamma^{*}\left(v_{j} \psi\right) \\
& =\sum_{i=1}^{r} \sum_{j=1}^{r} \Gamma^{*}\left(u_{i} \psi\right) X_{i} \Gamma^{*}\left(v_{i} s u_{j} \psi\right) X_{j} \Gamma^{*}\left(v_{j} \psi\right) \\
& =\sum_{i=1}^{r} \sum_{j=1}^{r} \Gamma^{*}\left(u_{i} \psi\right) X_{i} \Gamma^{*}\left(v_{i} \psi\right) \Gamma^{*}\left(s u_{j} \psi\right) X_{j} \Gamma^{*}\left(v_{j} \psi\right) \\
& =I_{n} \sum_{j=1}^{r} \Gamma^{*}\left(s u_{j} \psi\right) X_{j} \Gamma^{*}\left(v_{j} \psi\right) \\
& =\sum_{j=1}^{r} \Gamma^{*}\left(s u_{j} \psi\right) X_{j} \Gamma^{*}\left(v_{j} \psi\right) .
\end{aligned}
$$


For $s, t \in S$,

$$
\begin{aligned}
\Gamma(s) \Gamma(t) & =\Gamma(s) \sum_{j=1}^{r} \Gamma^{*}\left(t u_{j} \psi\right) X_{j} \Gamma^{*}\left(v_{j} \psi\right) \quad \text { by }(6.5) \\
& =\sum_{i=1}^{r} \sum_{j=1}^{r} \Gamma^{*}\left(u_{i} \psi\right) X_{i} \Gamma^{*}\left(v_{i} s \psi\right) \Gamma^{*}\left(t u_{j} \psi\right) X_{j} \Gamma^{*}\left(v_{j} \psi\right) \\
& =\sum_{i=1}^{r} \sum_{j=1}^{r} \Gamma^{*}\left(u_{i} \psi\right) X_{i} \Gamma^{*}\left(v_{i} s t u_{j} \psi\right) X_{j} \Gamma^{*}\left(v_{j} \psi\right) \\
& =\Gamma(s t) \quad \text { by (6.4). }
\end{aligned}
$$

Hence $\Gamma$ is a representation of $S$ of degree $n$ over $\Phi$ and, from its definition, the diagram (6.1) clearly commutes with $M$ replaced by $N$ and $V$ replaced by $U$. Further, from the definition of $\Gamma$, it is clear that $N \cdot \cdot U \subseteq V(\Gamma)$. On the other hand, if $N x \notin U$, the commutativity of (6.1) implies $\Gamma(n x) \neq 0$ for some $n \in N$ and so $x \notin V(\Gamma)$. Hence, to complete the proof of (d); we need only show that $\Gamma$ is basic.

Let $L$ be an ideal of $S$ such that $\Gamma(L) \neq 0$; that is, such that, $N L \nsubseteq U$. Then $N \cap L$ is an ideal of $N$ such that $N \cap L \nsubseteq U$. By the commutativity of (6.1) $\Gamma \mid N$ is basic and hence, if $\mathscr{V}$ is the underlying vector space for $\Gamma$,

$\mathscr{V}=[\mathscr{V} \Gamma(N \cap L)] \subseteq[\mathscr{V} \Gamma(\mathcal{L})]$ and $0 \neq v \Gamma(N \cap L) \subseteq \mathrm{v} \Gamma(L)$ for each nonzero $\mathrm{v} \in \mathscr{V}$.

Thus $\Gamma$ is basic.

(e) Let $\Delta$ be a representation of $S$ such that, for each $n \in N, \Delta(n)=\Gamma(n)$. Then, for $s \in S$,

$$
\begin{aligned}
\Delta(s) & =I_{n} \Delta(s)=\left[\sum_{1}^{+} \Gamma\left(u_{i}\right) X_{i} \Gamma\left(v_{i}\right)\right] \Delta(s) \\
& =\sum_{1}^{r} \Gamma\left(u_{i}\right) X_{i} \Delta\left(v_{i}\right) \Delta(s)=\sum_{1}^{r} \Gamma\left(u_{i}\right) X_{i} \Delta\left(v_{i} s\right)=\Gamma(s)
\end{aligned}
$$

since $v_{i} s \in N$ implies $\Delta\left(v_{i} s\right)=\Gamma\left(v_{i} s\right)$. Hence $\Gamma=\Delta$.

COROLlaRY 1. Let $\Gamma$ be a representation of a semigroup $S=S^{0}$ of degree $n$ over a field $\Phi$. Then $\Gamma$ is basic if and only if there exists a secondary pair $(U, N)$ such that $\Gamma \mid N$ induces a 0 -restricted homogeneous basic representation $\Gamma^{\prime}$ of $N / U$ such that the diagram

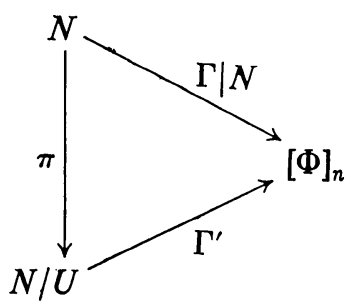


commutes. Equivalently, $\Gamma$ is basic if and only if $S / V$ is indecomposable at zero and $\Gamma \mid M$ induces a 0 -restricted basic representation $\Gamma^{\prime}$ of $M / V$ such that (6.6) commutes with $M$ replacing $N$ and $V$ replacing $U$.

COROLlary 2. Let $S=S^{0}$ be a semigroup and let $\Gamma, \Delta$ be basic representations of $S$. Then the following are equivalent :

(a) $\Gamma$ is equivalent to $\Delta$;

(b) $V(\Gamma)=V(\Delta), M(\Gamma)=M(\Delta)$ and the induced representations $\Gamma^{*}, \Delta^{*}$ are equivalent;

(c) $\Gamma$ is equivalent to $\Delta$ on some ideal $N$ such that $\Gamma(N) \neq 0$.

Proof. Clearly (a) implies (b) and (b) implies (c). Suppose that (c) holds and let $L=N \cap M(\Gamma)$. Since $\Gamma(N) \neq 0$ and $S / V(\Gamma)$ is indecomposable at zero, $\Gamma(L) \neq 0$ and further $(L \cap V(\Gamma), L)$ is a secondary pair of ideals of $S$. By hypothesis, there exists a nonsingular matrix $C$ such that, for each $x \in L, C^{-1} \Gamma(x) C=\Delta(x)$. Thus $C^{-1} \Gamma C$ is a representation of $S$ which extends $\Delta$; hence, by Theorem 6.2 (e), $\Delta=C^{-1} \Gamma C$ as required.

REMARK I. Let $S=S^{0}$ be a semigroup and let $(U, N)$ be a primary pair of ideals of $S$. Let $\Gamma$ be a representation of $S$ defined from a basic representation of $\mathscr{C}(N / U)$ as in Theorem 6.2. Then, for any $m, n \in N \backslash U,|\Gamma(m)|=|\Gamma(n)|$ so that $\Gamma(N)$ is homogeneous. On the other hand, since $(U, N)$ is a primary pair, it follows from Lemma 6.1 that $V(\Gamma)=U$. Hence, for $a \in S \backslash V(\Gamma)$, there exists $n \in N$ such that $a \notin \notin U$ and so $|\Gamma(n)|=|\Gamma(a n)| \leqq|\Gamma(a)|$; thus $N \subseteq M(\Gamma)$. In general, we cannot say that $N=M(\Gamma)$; in the case when $S$ is an inverse semigroup, it can be shown that $M(\Gamma)$ is the maximum ideal $L$ such that $(U, L)$ is a primary pair (see $\S 8$ ).

REMARK II. Let $S=S^{0}$ be a semigroup and let $U$ be an ideal of $S$ such that $S / U$ is indecomposable at zero. Suppose further that there is an ideal $N$ of $S$ which covers $U$. Then, by Theorem 6.2(II), the basic representations of $S$ with vanishing ideal $U$ are uniquely determined by their action on $N$. Now, for any $x \in N \backslash U$, we have $N=S^{1} x S^{1} \cup U$ and, if $I(x)=\left\{y \in S: S^{1} y S^{1} \subseteq S^{1} x S^{1}\right\},\left(I(x), S^{1} x S^{1}\right)$ is a secondary pair with $I(x)=S^{1} x S^{1} \cap U$. Thus the basic representations of $S$, with vanishing ideal $U$, are uniquely determined by basic representations of the principal factor $J(x)=S^{1} x S^{1} / I(x)$ (since $\left(I(x), S^{1} x S^{1}\right)$ is a secondary pair, $J(x)$ is 0 -simple). Hence, if $S$ obeys the minimal condition $M_{J}$ on principal two-sided ideals, the basic representations of $S$ are obtained uniquely as extensions of the basic representations of the principal factors of $S$. That is, the situation, for basic representations is exact analogy with the situation, discovered by Munn [10], for irreducible representations.

REMARK III. Let $S=S^{0}$ be a semigroup and let $(U, N)$ be a secondary pair of ideals of $S$. Let $\Gamma^{*}$ be a homogeneous representation of $N$ with vanishing ideal $U$. Then, in order that we should be able to extend $\Gamma^{*}$ to a representation $\Gamma$ of $S$ as in the proof of Theorem 6.2, it is sufficient that there exist $u_{i}, v_{i} \in N, X_{i} \in[\Phi]_{n}$ such that

$$
\sum \Gamma^{*}\left(u_{i}\right) X_{i} \Gamma^{*}\left(v_{i}\right)=I_{n}
$$


that is, it is sufficient that $I_{n} \in\left[\Gamma^{*}(N)[\Phi]_{n} \Gamma^{*}(N)\right]$. Suppose however that the latter condition is satisfied and let $\mathbf{v}$ be a vector in the underlying vector space $\mathscr{V}$. Then $\mathbf{v} I_{n} \neq 0$ and hence $\mathbf{v} \Gamma^{*}(n) \neq 0$ for some $n \in N \backslash U$. On the other hand, if $\mathscr{V} \Gamma^{*}(N)$ is contained in the subspace $\mathscr{Y}$ of $\mathscr{V}$ then $\Gamma^{*}(N)[\Phi]_{n} \Gamma^{*}(N) \subseteq \mathscr{Y}$. Hence, since $I_{n} \in\left[\Gamma^{*}(N)[\Phi]_{n} \Gamma^{*}(N)\right], \mathscr{Y}=\mathscr{V}$. Therefore $\Gamma^{*}$ is basic and, from Theorem 6.2(d), the same is true of $\Gamma$.

As a corollary to these remarks, we have the following characterisation of basic representations.

THEOREM 6.3. Let $S=S^{0}$ be a semigroup and let $\Gamma$ be a representation of $S$ of degree $n$ over a field $\Phi$. Then $\Gamma$ is basic if and only if

(a) $(V, M)$ is a primary pair;

(b) $I_{n} \in\left[\Gamma(M)[\Phi]_{n} \Gamma(M)\right]$.

If $\Gamma$ is an irreducible representation of a semigroup $S=S^{0}$ then, since $\Gamma(N)$ is irreducible for each ideal $N$ of $S$ not contained in $V(\Gamma), \Gamma$ is easily seen to be basic. Hence Theorem 6.2 is applicable to the irreducible representations of $S$. However, it follows, from [3, Lemma 5.32], that we can choose the elements which satisfy (6.2) in a particularly simple manner. Theorem 6.4 describes the structure of all irreducible representations of $S$; although it is a corollary to Theorem 6.2 , we state it in full here because of the importance of irreducible representations. (The proof follows easily from Theorem 6.2 and Theorem 4.5.)

THEOREM 6.4. Let $S=S^{0}$ be a semigroup and let $\Phi$ be a field.

(I) Let $\Gamma$ be an irreducible representation of $S$ of degree $n$ over $\Phi$.

(a) $(V, M)$ is a primary pair and $\Gamma$ induces a (unique) 0-restricted irreducible representation $\Gamma^{*}$ of $\mathscr{C}(M / V)$ such that the diagram

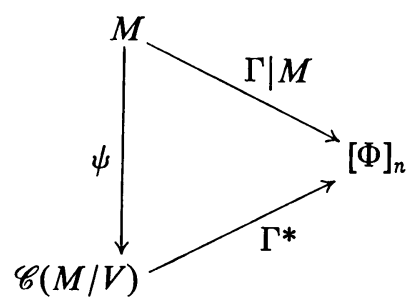

commutes, where $\psi=f \eta$, $f$ the natural homomorphism of $M$ onto $M / V$ and $\eta$ the natural 0 -restricted homomorphism of $M / V$ into $\mathscr{C}(M / V)$.

(b) There exist elements $\alpha_{i} \in \Phi, u_{i} \in M, i=1,2, \ldots, r$ such that

(c) For each $s \in S$,

$$
\sum_{1}^{r} \alpha_{i} \Gamma^{*}\left(u_{i} \psi\right)=I_{n}
$$

$$
\Gamma(s)=\sum_{1}^{r} \alpha_{i} \Gamma^{*}\left(u_{i} s \psi\right) .
$$


(II) Let $(U, N)$ be a secondary pair of ideals of $S$ and let $\Gamma^{*}$ be a 0 -restricted irreducible representation of $\mathscr{C}(N / U)$ of degree $n$ over $\Phi$.

(d) There exist elements $\alpha_{i} \in \Phi, u_{i} \in N, i=1,2, \ldots, r$, such that (6.7) is satisfied. For these elements, the mapping $\Gamma$ defined by (6.8) is an irreducible representation of $S$ of degree $n$ over $\Phi$ such that (6.1) commutes with $M$ replaced by $N$ and $V$ replaced by $U$. Further $V(\Gamma)=N . \cdot U$.

(e) If $\Delta$ is any representation of $S$, of degree $n$ over $\Phi$, such that $\Delta|N=\Gamma| N$ then $\Delta=\Gamma$; in particular, $\Gamma$ is independent of the particular choice of elements satisfying (6.7).

7. Basically reducible representations. Let $S=S^{0}$ be a semigroup and let $\Gamma$ be a representation of $S$ of degree $n$ over a field $\Phi$. Then $\Gamma$ is fully basically reducible if each nonnull indecomposable representation of $\Gamma$ is basic. Clearly any fully reducible representation of $S$ is fully basically reducible.

THEOREM 7.1. Any basic representation of a semigroup $S=S^{0}$, of degree $n$ over a field $\Phi$, is fully basically reducible.

Proof. We need only show that if $\Gamma$ decomposes into the direct sum of two representations $\Gamma_{1}$ and $\Gamma_{2}$ then each of these is basic. Let $\mathscr{V}_{1}$ and $\mathscr{V}_{2}$ be the underlying vector spaces for $\Gamma_{1}$ and $\Gamma_{2}$; then $\mathscr{V}=\mathscr{V}_{1} \oplus \mathscr{V}_{2}$ is an underlying vector space for $\Gamma$.

Let $N$ be an ideal of $S$ such that $\Gamma(N) \neq 0$. Let $\mathbf{v} \in \mathscr{V}_{1} \mid 0$; then $\mathbf{v} \in \mathscr{V}$ and so there exists $n \in N$ such that $\mathbf{v} \Gamma(n) \neq 0$. But, since $\mathscr{V}_{1} \Gamma_{2}(S)=0$, this implies $\mathscr{V} \Gamma_{1}(n) \neq 0$. If $\mathscr{Y}$ is a subspace of $\mathscr{V}_{1}$ such that $\mathscr{V}_{1} \Gamma_{1}(N) \subseteq \mathscr{Y}$, then

$$
\mathscr{V} \Gamma(N)=\left\{\mathscr{V}_{1} \Gamma_{1}(n)+\mathscr{V}_{2} \Gamma_{2}(n): n \in N\right\} \subseteq \mathscr{Y} \oplus \mathscr{V}_{2}
$$

Hence, since $\Gamma$ is basic, $\mathscr{Y}=\mathscr{V}_{1}$. Thus $\Gamma_{1}$ is basic and similarly so is $\Gamma_{2}$.

COROLlARY 1 (TO THE PROOF). Each indecomposable constituent of a basic representation $\Gamma$ has vanishing ideal $V(\Gamma)$.

CoRollary 2. A representation of $S$ of degree $n$ over a field $\Phi$ is fully basically reducible if and only if it is the direct sum of basic representations.

Following Corollary 2, we shall drop the unwieldy term "fully basically reducible" and shall use in its stead the term basically reducible. Our next theorem gives necessary and sufficient conditions on a representation in order that it should be basically reducible. For the proof of the theorem, we require two lemmas which will also be used to give sufficient conditions on a semigroup in order that every representation over a field $\Phi$ should be basically reducible.

LEMMA 7.2. Let $S=S^{0}$ be a semigroup and let $\Gamma$ be a 0 -restricted representation of $S$ of degree $n$ over a field $\Phi$. Let $\bar{M}$ be a nonzero ideal of $S$ and suppose that, $\Gamma \mid \bar{M}$ decomposes into the direct sum of two representations $\Gamma_{1}$ and $\Gamma_{2}$ of degrees $r$ and $n-r$ 
respectively. Suppose further that $\Gamma_{1}$ is basic. Then $\Gamma$ decomposes into the direct sum of two representations $\Gamma_{1}^{\prime}$ and $\Gamma_{2}^{\prime}$ of $S$ where $\Gamma_{i}^{\prime} \mid \bar{M}=\Gamma_{i}, i=1,2$.

Proof. Let $\mathscr{Y}$ be the subspace of $[\Phi]_{n}$ of all matrices of the form

$$
\left[\begin{array}{ll}
X & 0 \\
0 & 0
\end{array}\right], \quad \text { where } X \text { is } r \times r \text {. }
$$

Then $[\Gamma(\bar{M}) \mathscr{Y} \Gamma(\bar{M})] \subseteq \mathscr{Y}$ and, since by hypothesis $\Gamma_{1}$ is basic,

$$
E=\left[\begin{array}{ll}
I_{r} & 0 \\
0 & 0
\end{array}\right] \in[\Gamma(\bar{M}) \mathscr{Y} \Gamma(\bar{M})] .
$$

For each $s \in S$, let $\Gamma(s)$ be partitioned as

$$
\left[\begin{array}{ll}
\Gamma_{1}^{\prime}(s) & \Gamma_{12}^{\prime}(s) \\
\Gamma_{21}^{\prime}(s) & \Gamma_{2}^{\prime}(s)
\end{array}\right]
$$

where $\Gamma_{1}^{\prime}(s)$ is $r \times r$. Since $\bar{M}$ is an ideal of $S$,

$$
\Gamma(S)[\Gamma(\bar{M}) \mathscr{Y} \Gamma(\bar{M})] \text { and }[\Gamma(\bar{M}) \mathscr{Y} \Gamma(\bar{M})] \Gamma(S)
$$

are both contained in $\mathscr{Y}$ and hence, in particular, $E \Gamma(S)$ and $\Gamma(s) E$ are in $\mathscr{Y}$. Thus $\Gamma_{12}^{\prime}(s)=0, \Gamma_{21}^{\prime}(s)=0$ for each $s \in S$ and so we have the result.

Lemma 7.3. Let $S=S^{0}$ be a semigroup and let $M$ be a nonzero ideal of $S$. If $M=0\left\{M_{i}: i \in I\right\}$ where each $M_{i}$ has zero biannihilator then each $M_{i}$ is an ideal of $S$.

Proof. Let $m_{i} \in M_{i} \mid 0$ and let $a \in S$. Then $a m_{i} \in M$ since $M$ is an ideal of $S$. If $a m_{i} \in M_{j} \mid 0$ then, since $B\left(M_{j}\right)=0$, there exists $m_{j} \in M_{j}$ such that $a m_{i} m_{j} \neq 0$. Thus, since $M=0\left\{M_{i}: i \in I\right\}, i=j$. Hence $a M_{i} \subseteq M_{i}$ and $M_{i}$ is a left ideal of $S$. Similarly it is a right ideal; thus an ideal.

Following the terminology introduced in $\S 2$, we shall call a representation $\Gamma$ of a semigroup $S=S^{0}$, weakly basic if,

(a) for each $\mathbf{v} \in \mathscr{V} \mid 0, \mathbf{v} \Gamma(S) \neq 0$;

(b) $[\mathscr{V} \Gamma(S)]=\mathscr{V}$;

where $\mathscr{V}$ is the underlying vector space for $\Gamma$.

THEOREM 7.4. Let $S=S^{0}$ be a semigroup and let $\Gamma$ be a representation of $S$ of degree $n$ over a field $\Phi$. Then $\Gamma$ is basically reducible if and only if the proper part of $\Gamma \mid N$ is weakly basic for each ideal $N$ of $S$ which is not contained in $V(\Gamma)$.

Proof. Suppose that $\Gamma$ is basically reducible so that it is the direct sum of basic representations $\Gamma_{i}$. Let $\Gamma_{N}$ be the direct sum of those $\Gamma_{i}$ such that $\Gamma_{i}(N) \neq 0$; $i=1,2, \ldots, r$ say. Then $\Gamma \mid N$ clearly decomposes into $\Gamma_{N}$ and a null representation. We show that $\Gamma_{N}$ is weakly basic; it is then certainly proper so that we have the necessity of the conditions.

Let $\mathscr{V}$ be the underlying vector space for $\Gamma_{N}$ and let $\mathbf{v} \in \mathscr{V}$. Then $\mathbf{v}=\mathbf{v}_{1}+\cdots+\mathbf{v}_{r}$ where $\mathbf{v}_{i} \in \mathscr{V}_{i}$ the underlying vector space for $\Gamma_{i}, 1 \leqq i \leqq r$. If $\mathbf{v} \Gamma_{N}(N)=0$ then each 
$\mathbf{v}_{i} \Gamma_{i}(N)=0$ and hence, since each $\Gamma_{i}$ is basic and no $\Gamma_{i}(N)=0, \mathbf{v}_{i}=0,1 \leqq i \leqq r$. This implies $\mathbf{v}=0$ and so (a) of the definition of weakly basic holds.

Let $\mathscr{Y}$ be a subspace of $\mathscr{V}$ and suppose that $\mathscr{V} \Gamma_{N}(N) \subseteq \mathscr{Y}$. Then $\mathscr{V}_{i} \Gamma_{i}(N)=$ $\mathscr{V}_{i} \Gamma_{N}(N) \subseteq \mathscr{Y}$ and hence, since each $\Gamma_{i}$ is basic, $\mathscr{V}_{i} \subseteq \mathscr{Y}$. Thus $\mathscr{V}=\mathscr{V}_{i} \oplus \cdots \oplus \mathscr{V}_{r} \subseteq \mathscr{Y}$; hence (b) holds and so $\Gamma_{N}$ is weakly basic.

Conversely, it follows, as in the proof of Theorem 7.1, that if $\Gamma$ has the properties mentioned above so has each indecomposable constituent of $\Gamma$. Hence we need only show that an indecomposable representation, with these properties, is basic. We can also assume, without loss of generality, that $\Gamma$ is 0 -restricted.

Let $\mathscr{V}$ be the underlying vector space for $\Gamma_{M}$ the proper part of $\Gamma \mid M$ and let $a \in M \backslash 0$. Then there exists $\mathbf{v} \in \mathscr{V}$ such that $\mathbf{v} \Gamma_{M}(a) \neq 0$. By (a) of the definition of weakly basic, there exists $m \in M$ such that $\mathrm{v} \Gamma_{M}(a) \Gamma_{M}(m) \neq 0$; thus $a m \neq 0$. By (b), there exists $n \in M$ such that, for some $\mathbf{u} \in \mathscr{V}, \mathbf{u} \Gamma_{M}(n) \notin$ null space $\Gamma_{M}(a)$. Then $\mathbf{u} \Gamma_{M}(n) \Gamma_{M}(a) \neq 0$; thus $n a \neq 0$. Since $M$ is categorical at zero, nam $\neq 0$; thus $M a M$ $\neq 0$. Repeating the argument with $M a M$ in place of $M$, we find that $M a M \cdot M a M$ $\neq 0$; thus $a M a \neq 0$. Hence $M$ has no nonzero nilpotent ideals and thus, by $[4$, Corollary 5.13], $M=0\left\{M_{i}: i \in I\right\}$ where each $M_{i}$ is 0 -primary. Therefore, by Lemma 7.3, each $M_{i}$ is an ideal of $S$.

Pick $1 \in I$ and let $\Gamma_{1}$ denote the proper part of $\Gamma \mid M_{1}$; then $\Gamma_{1}$ is homogeneous and hence, from Theorem 3.11, $\Gamma \mid M_{1}$ satisfies the hypothesis of Lemma 7.2. Thus, if $M_{1} \neq M$ or $\Gamma \mid M_{1}$ is not proper, $\Gamma$ is decomposable. Hence $M=M_{1}$ and $\Gamma \mid M=\Gamma_{1}$; thus, by Theorem 3.11, $\Gamma \mid M$ is basic. Since $M$ is thus 0 -primary, it follows from Theorem 6.2, Corollary 1 , that $\Gamma$ is basic.

COROLlaRY. Let $S=S^{0}$ be a semigroup and let $\Gamma$ be a representation of $S$ of degree $n$ over a field $\Phi$. If, for each ideal $N$ of $S$ which is not contained in $V(\Gamma)$, the proper part of $\Gamma \mid N$ has no null irreducible constituents, then $\Gamma$ is basically reducible.

Proof. Suppose $\mathbf{v} \Gamma_{N}=0$, where $\Gamma_{N}$ is the proper part of $\Gamma \mid N$, then since $\Gamma_{N}$ has no null irreducible constituents, $\mathbf{v}=0$. Similarly, it is impossible that $\left[\mathscr{V} \Gamma_{N}(N)\right] \subset \mathscr{V}$.

It is shown in [8] that, for finite semigroups, the converse of the above corollary also holds.

We define a partial order on the set $\mathscr{X}(S)$ of all secondary pairs of ideals of a semigroup $S=S^{0}$ as follows:

$\left(U^{\prime}, N^{\prime}\right) \leqq(U, N)$ if and only if $N^{\prime} \subseteq N$ and $U^{\prime}=N^{\prime} \cap U$; thus $\left(U^{\prime}, N^{\prime}\right) \leqq(U, N)$ if and only if the diagram

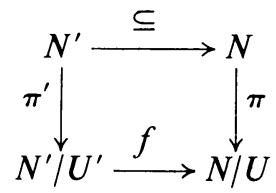

commutes, where $\pi$ and $\pi^{\prime}$ are the natural homomorphisms and $f$ is 0 -restricted. A subset $\mathscr{X}^{\prime}$ of $\mathscr{X}(S)$ is a coinitial subset if, for each pair $(U, N) \in \mathscr{X}(S)$, there exists 
$\left(U^{\prime}, N^{\prime}\right) \in \mathscr{X}^{\prime}$ such that $\left(U^{\prime}, N^{\prime}\right) \leqq(U, N)$. If $\mathscr{X}^{\prime}$ is a coinitial subset of $\mathscr{X}(S)$, we shall call the Rees factor semigroups $N / U$ where $(U, N) \in \mathscr{X}^{\prime}$, secondary factors in $\mathscr{X}^{\prime}$.

EXAMPLE. Let $S=S^{0}$ be a semigroup which obeys the minimal condition $M_{J}$ on two-sided principal factors. Let $\mathscr{X}^{\prime}$ be the set of all pairs $\left(I(x), S^{1} x S^{1}\right)$, where $I(x)=\left\{y \in S: S^{1} y S^{1} \subset S^{1} x S^{1}\right\}$, such that the principal factor $J(x)=S^{1} x S^{1} / I(x)$ is 0 -primary. Then $\mathscr{X}^{\prime}$ is the unique minimal coinitial subset of $\mathscr{X}(S)$.

Recall that a semigroup $S=S^{0}$ is quasi-simple over a field $\Phi$ if each indecomposable representation of $S$ over $\Phi$ is basic.

THeOREM 7.5. Let $S=S^{0}$ be a semisimple semigroup and let $\mathscr{X}^{\prime}$ be a coinitial subset of $\mathscr{X}(S)$. Let $\Phi$ be a field; if each proper 0-restricted homogeneous representation over $\Phi$ of each secondary factor in $\mathscr{X}^{\prime}$ is basic then $S$ is quasi-simple over $\Phi$.

Proof. We need only show that each indecomposable representation of $S$ over $\Phi$ is basic; let $\Gamma$ be such. Since $S$ is semisimple, $M / V$ has no nonzero nilpotent ideals and further, since $\Gamma(M)$ is homogeneous, $M / V$ is categorical at zero. Hence, by [4, Corollary 5.13], $M / V=0\left\{M_{i} / V: i \in I\right\}$ where each $\left(V, M_{i}\right)$ is a secondary pair of ideals of $S$.

Let $(U, N) \in \mathscr{X}^{\prime}$ be such that $(U, N) \leqq\left(V, M_{i}\right)$. Then, by the hypothesis on $\mathscr{X}^{\prime}$, we can apply Lemma 7.2 to $\Gamma$ and $N$. Hence, if $\Gamma \mid N$ is not proper, $\Gamma$ decomposes which is a contradiction to its choice. Thus $\Gamma \mid N$ is proper and hence $\Gamma$ extends the basic representation $\Gamma \mid N$ of $N$, which has vanishing ideal $U$. By Corollary 1 to Theorem 6.2, this ensures that $\Gamma$ is basic as required.

Corollary. Let $S=S^{0}$ be a semisimple semigroup which obeys the minimal condition $M_{J}$ on two-sided principal ideals. If each proper representation of each principal factor of $S$ over a field $\Phi$ is basic, then $S$ is quasi-simple over $\Phi$.

In [8] it is shown that the converse of this corollary holds for finite semigroups.

The proof of the following theorem differs from that of Theorem 7.5 only in that we use Theorem 6.4 instead of Theorem 6.2.

THEOREM 7.6. Let $S=S^{0}$ be a semisimple semigroup and let $\mathscr{X}^{\prime}$ be a coinitial subset of $\mathscr{X}(S)$. Let $\Phi$ be a field and suppose that each 0-restricted homogeneous representation, over $\Phi$, of each secondary factor in $\mathscr{X}^{\prime}$ is completely reducible. Then each representation of $S$ over $\Phi$ is completely reducible.

COROLlaRY (MUNN [10]). Let $S=S^{0}$ be a semisimple semigroup which obeys the minimal condition $M_{J}$ on principal two-sided ideals. If each representation of each principal factor of $S$ over a field $\Phi$ is completely reducible, then each representation of $S$ over $\Phi$ is completely reducible.

8. Inverse semigroups. The theory given in previous sections applies, in particular, to inverse semigroups. For, clearly, any inverse semigroup $S=S^{0}$ is semisimple and further, if $(U, N) \in \mathscr{X}(S), \mathscr{C}(N / U)$ is a Brandt semigroup thus each of its 
proper representations is basic; [1, Theorem 8.2]. Hence each proper 0-restricted homogeneous representation of $N / U$ is basic. By Theorem 7.5, we have the following theorem, which can also be deduced from the results of Preston [14].

THEOREM 8.1. If $S=S^{0}$ is an inverse semigroup and $\Phi$ is any field then $S$ is quasi-simple over $\Phi$.

The structure of the basic representations of an inverse semigroup is much simpler than the structure of basic representations of an arbitrary semigroup. Such representations are described in the following theorem, the proof of which is entirely analogous to that of Munn's theorem on irreducible representations of inverse semigroups [13].

THEOREM 8.2. Let $S=S^{0}$ be an inverse semigroup and let $\Phi$ be a field.

(I) Let $\Gamma$ be a basic representation of $S$ of degree $n$ over $\Phi$.

(a) $(V, M)$ is a primary pair and is maximal in $\mathscr{X}(S)$. Further $\mathscr{C}(M / V)$ has only a finite number of nonzero idempotents, $e_{1} \psi, \ldots, e_{r} \psi$ where $r$ divides $n . \Gamma$ induces a unique basic representation $\Gamma^{*}$ of $\mathscr{C}(M / V)$ such that the diagram

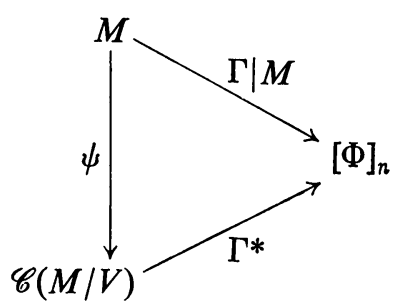

commutes, where $\psi=\pi \eta, \pi$ the natural homomorphism of $M$ onto $M / V$ and $\eta$ the natural 0-restricted homomorphism of $M / V$ into $\mathscr{C}(M / V)$.

(b)

$$
\sum_{1}^{r} \Gamma^{*}\left(e_{i} \psi\right)=I_{n}
$$

(c) For each $s \in S$,

$$
\Gamma(s)=\sum_{1}^{r} \Gamma^{*}\left(e_{i} s \psi\right)
$$

(II) Let $(U, N)$ be maximal in $\mathscr{X}(S)$ and suppose that $\mathscr{C}(M / V)$ has only a finite number of idempotents $e_{1} \psi, \ldots, e_{r} \psi$. Then $\mathscr{C}(M / V)$ has a proper representation $\Gamma$ of degree $n$ over $\Phi$ where $n$ is any integral multiple of $r$.

(d) (8.2) is satisfied and the mapping $\Gamma$ defined by (8.3) is a basic representation of $S$ of degree $n$ over $\Phi$ such that the diagram (8.1) commutes with $M$ replaced by $N$ and $V$ replaced by $U$. Further $V(\Gamma)=U$ and $M(\Gamma)=N$.

(e) $\Gamma$ is indecomposable if and only if the same is true of $\Gamma^{*}$; it is irreducible if and only if $\Gamma^{*}$ is irreducible. 
Theorem 8.1 of [1] and Theorems 8.1 and 8.2 here give a complete description of all representations of an arbitrary inverse semigroup. Further, from Munn [12], if $(U, N)$ is maximal in $\mathscr{X}(S), \mathscr{C}(N / U)$ is $N / \sigma$ where

$$
\sigma=\{(x, y) \in N \times N: x z=y z \notin U \text { for some } z \in N\} \cup U \times U
$$

$\sigma$ is a congruence on $N$.

\section{REFERENCES}

1. A. H. Clifford, Matrix representations of completely simple semigroups, Amer. J. Math. 64 (1942), 327-342.

2. —_- Basic representations of completely simple semigroups, Amer. J. Math. 82 (1960), $430-434$.

3. A. H. Clifford and G. B. Preston, Algebraic theory of semigroups, Vol. I, Math. Surveys, No. 7, Amer. Math. Soc., Providence, R. I., 1961.

4. G. Lallement and M. Petrich, Décompositions I-matricelle d'un demi-groupe, J. Math. Pures Appl. 45 (1966), 67-117.

5. - - Irreducible matrix representations of finite semigroups, (to appear).

6. D. B. McAlister, Matrix representations of semigroups, Glasgow Math. J. (1) 8 (1967), 1-13.

7. —, A homomorphism theorem for semigroups, J. London Math. Soc. 43 (1968), 355-366.

8. - Basic representations of finite semigroups, Czechoslovak Math. J. (to appear).

9. W. D. Munn, Matrix representations of semigroups, Proc. Cambridge Philos. Soc. 53 (1957), 145-152.

10. - Irreducible matrix representations of semigroups, Quart. J. Math. Oxford Ser. (2) 11 (1960), 295-309.

11. - A class of irreducible matrix representations of an arbitrary inverse semigroup, Proc. Glasgow Math. Assoc. 5 (1961), 41-48.

12. - Brandt congruences on inverse semigroups, Proc. London Math. Soc. (3) 14 (1964), 154-164.

13. - Matrix representations of inverse semigroups, Proc. London Math. Soc. (3) 14 (1964), 165-181.

14. G. B. Preston, Matrix representations of inverse semigroups, J. Austral. Math. Soc. (to appear).

TULANE UNIVERSITY,

New OrLeans, Louisiana 